Cahiers de recherches médiévales

\title{
Les venins imaginaires
}

Le poison dans la Suède médiévale $\left(\mathrm{XIII}^{\mathrm{e}}-\mathrm{XV}^{\mathrm{e}}\right.$ siècle $)$

\section{Corinne Péneau}

\section{(2) OpenEdition \\ 1 Journals}

Édition électronique

URL : https://journals.openedition.org/crm/11506

DOI : $10.4000 / \mathrm{crm} .11506$

ISSN : 1955-2424

Éditeur

Honoré Champion

\section{Édition imprimée}

Date de publication : 15 juin 2009

Pagination : 71-102

ISSN : 1272-9752

\section{Référence électronique}

Corinne Péneau, «Les venins imaginaires », Cahiers de recherches médiévales [En ligne], 17 | 2009, mis en ligne le 15 juin 2012, consulté le 15 décembre 2022. URL : http://journals.openedition.org/crm/ 11506 ; DOI : https://doi.org/10.4000/crm.11506

Ce document a été généré automatiquement le 15 décembre 2022.

Tous droits réservés 


\title{
Les venins imaginaires
}

\author{
Le poison dans la Suède médiévale $\left(\mathrm{XIII}^{\mathrm{e}}-\mathrm{XV}^{\mathrm{e}}\right.$ siècle)
}

\section{Corinne Péneau}

1 Grâce aux sagas, le poison et son imaginaire magique ${ }^{1}$ sont bien connus dans le monde norrois $^{2}$ : l'épisode où Egill Skalla-Grímsson échappe à une tentative d'empoisonnement de la reine Gunnhildr est célèbre. C'est grâce à sa connaissance de la magie qu'il déjoue le piège : usant de runes, qu'il grave sur la corne à boire et arrose de son sang, il réussit à faire voler en éclats la corne, ce qui lui révèle la présence du poison et lui sauve la $v^{2} e^{3}$. Des membres de la famille royale, comme Halfdan le Noir ${ }^{4}$, fils du roi Harald à la Belle Chevelure, peuvent aussi être les victimes malheureuses des poisons.

2 Si l'on en croit Saxo Grammaticus, la Suède était une contrée de monstres venimeux, comme en témoignent les deux serpents élevés par la fille du roi Herothus que le roi danois Regnerus vainquit grâce à une tunique gelée qui le protégea des jets continus de venins ${ }^{5}$. Mais, côté suédois, les sources ne sont pas aussi fournies en épisodes dramatiques. On aurait pu s'attendre à ce que cette monarchie élective soit le théâtre d'empoisonnements politiques ${ }^{6}$. Il n'en a rien été : rois, régents et prétendants à la couronne ont été écartés ou sont morts sans l'aide des poisons et il semble qu'euxmêmes n'y aient pas eu recours. L'historien peut toutefois glaner quelques cas intéressants : l'affirmation précédente supporte, en effet, deux petites exceptions et les Suédois n'ignorèrent ni le poison de la rumeur, ni les usages politiques de l'accusation d'empoisonnement ${ }^{7}$.

Le poison est loin d'être l'arme qui fit le plus scandale dans la Suède médiévale : pour la période que nous avons retenue, deux épisodes soulevèrent l'indignation et coûtèrent au roi son trône. Le premier est le célèbre banquet de Nyköping, qui, pour être un banquet mortel, ne fut pas un banquet empoisonné. En décembre 1317, le roi Birger Magnusson, qui ne régnait alors que sur une partie du royaume de Suède, invita ses frères, les ducs Erik et Valdemar, qui se partageaient l'autre moitié du royaume, à un banquet dans sa forteresse de Nyköping : dans la nuit, les ducs furent arrêtés, jetés en prison et ils moururent de faim au début de l'année suivante. L'épisode fut largement connu ${ }^{8}$. L'Erikskrönika, chronique rédigée au début du règne du roi Magnus, le fils du duc Erik et le successeur du roi Birger, consacre une longue description à l'épisode et 
on trouve sans doute son écho dans l'adaptation suédoise du De regimine principum de Gilles de Rome réalisée quelques années plus tard. Gilles de Rome précisait au sujet des tyrans : proprios fratres et nimia sibi consanguinitate conjunctos venenant et perimunt ${ }^{9}$. La version suédoise complète la liste et semble, comme l'a souligné Knut Frederik Söderwall, donner au tyran un visage bien identifié :

Il cherche, par la violence et de mauvais conseils, à les perdre par tous les moyens possibles, l'empoisonnement, la ruine, la prison et une mort infamante. ${ }^{10}$

Il est possible que maître Mathias dans l'Homo conditus fasse aussi référence à cet épisode lorsque, dans le chapitre consacré à l'horreur de l'homicide, il s'écrit :

Drachonibus vtique seuior est, o homocida! Venenosissimi drachones, licet crudelissimi sint, tamen sibi, socios scilicet et fratres, diligunt ${ }^{11}$.

5 Bien que le terme fratres puisse être interprété avec sa nuance chrétienne, l'expression socios et fratres indique qu'il faut sans doute lui donner son sens propre: pire qu'un dragon venimeux, Birger n'aura pas eu besoin des poisons pour faire scandale.

L'autre épisode qui révolta les Suédois fut le Bain de Sang de Stockholm, en novembre 1520. Le roi Christian II et l'archevêque Gustav Trolle se vengeaient ainsi des partisans de Sten Sture le Jeune, mort en février, mais dont le corps fut pour l'occasion tiré de la tombe et exposé avec les quatre-vingt deux victimes de ce massacre, évêques, nobles et bourgeois réunis. C'est donc l'épée qui précipita la fin de l'Union de Kalmar.

7 Le poison ne fut pourtant pas absent entre le xIII ${ }^{e}$ et le début du $\mathrm{XVI}^{\mathrm{e}}$ siècle. Ainsi, au XIV ${ }^{e}$ siècle, l'adaptateur anonyme du miroir de Gilles de Rome a conservé le sens du verbe venenare, en le rendant par le substantif forgärning, un mot à la fois plus précis, puisqu'il désigne le crime, et plus ambigu, puisqu'il signifie le crime par empoisonnement, mais aussi tout crime qui n'est pas lié à une forme de violence extérieure $^{12}$, en particulier toute pratique liée à la magie ${ }^{13}$. Le vocabulaire des poisons apparaît même assez riche puisque l'on trouve, dans le même sens que forgärning, le substantif ogärning ${ }^{14}$. Le mot pour désigner le venin de l'animal est eter. Le poison, au sens de liquide empoisonné, se dit eters drykker, et, à la fin du Moyen Âge, on trouve les substantifs forgift, ou encore forgiftils ${ }^{15}$. Le verbe emprunté à l'allemand, qui a donné förgifta en suédois moderne, est utilisé pour "empoisonner", mais l'on trouve aussi dans ce sens forgiva dans les textes historiographiques et littéraires. En revanche, le verbe firigcra ne prend que rarement le sens de "tuer par magie ou par poison ${ }^{16}$ ». Dans les lois, le pluriel forgärningar qui désigne de manière générale les maléfices, les procédés utilisés pour nuire à une personne, peut aussi prendre le sens particulier de poison $^{17}$. Ces frontières floues entre la sorcellerie et l'usage des poisons, qui supposent à la fois une connaissance spécifique et des buts mauvais, ne sont pas propres au monde nordique : comme le rappelle Franck Collard, le crimen veneficii apparaît dans le droit romain étroitement lié à la magie et les lois médiévales héritent directement ou non de cette confusion ${ }^{18}$.

8 Les archives judiciaires manquent pour retracer une véritable histoire du poison en Suède ${ }^{19}$, mais, comme nous l'évoquerons dans le cadre de ce petit article, les venins ont été pensés dans les lois et distillés dans les chroniques ou dans les textes politiques, offerts à l'opinion lors de moments de crise. C'est cette histoire, non des faits, mais des perceptions d'un type particulier de crime, que nous proposons de retracer. 


\section{« Tu m’as empoisonné ! » - Le poison dans les lois suédoises}

9 Les lois suédoises forment un corpus d'une quinzaine de textes. Certaines sont conservées sous forme fragmentaire (c'est le cas de la Loi du Småland dont on ne connaît que le Code de l'Église) et d'autres ne concernent que des espaces limités, comme l'ancienne législation sur les villes appelée Bjärköarätten, sans doute appliquée dès la seconde moitié du XIII siècle, mais conservée dans un manuscrit tardif datant de 1345. Les lois furent rédigées dans le cadre des provinces, les plus importantes formant une lagsaga, une circonscription juridique représentée par une assemblée des hommes libres (un thing). Cette assemblée, dont émanait la loi, était dirigée par un lagman, chargé de réciter la loi, de l'appliquer et, parfois, de participer à sa rédaction. À partir de 1250, l'influence de l'Église, de l'aristocratie et du pouvoir royal est importante dans les lois. Le roi peut légifèrer et promulguer officiellement la loi comme ce fut le cas en 1296 pour la Loi d'Uppland et en 1327 pour la Loi du Södermanland. On distingue deux principaux groupes de lois provinciales, les lois du Götaland, au sud de la Suède, et celles du Svealand, au centre du royaume. L'Ancienne Loi du Västergötland, qui n'est connue que par un unique manuscrit datant des années 1280 , fut sans doute rédigée à partir des années 1220. Une autre version plus récente de la loi fut composée vers la fin du XIII ${ }^{\mathrm{e}}$ siècle. Ce fut sans doute également à la fin de ce siècle ${ }^{20}$ que fut rédigée la Loi d'östergötland qui s'appliquait aussi dans des régions du Småland et sur l'île d'öland. Quant aux lois du Svealand, la plus ancienne est sans doute la Loi de Dalécarlie $e^{21}$, qui présente des traits archaïques, mais la plus importante reste la Loi d'Uppland, qui influença fortement les autres lois rédigées dans la première moitié du XIV siècle, en particulier, dans les années 1320, la Loi du Hälsingland, qui s'appliquait aussi au nord de la Suède et dans l'actuelle Finlande, ainsi que, dans une moindre mesure, la Loi du Västmanland et celle du Södermanland. Vers le milieu du xive siècle, sous le règne du roi Magnus Eriksson, une commission, à l'œuvre dès 1347, fut chargée de rédiger une loi valable dans l'ensemble du royaume. Cette Loi nationale (landslag), qui intégrait un bon nombre d'ordonnances royales, ne fut jamais officiellement promulguée, mais elle fut très largement diffusée et utilisée dès la seconde moitié du siècle, sans toutefois remplacer complètement les lois provinciales. Dans les années 1350 fut également rédigée une Loi urbaine (stadslag) destinée à s'imposer à toutes les villes du royaume. Imprimée en 1618, cette loi ne fut remplacée qu'en 1734-1736. En 1442, la Loi nationale, sous une forme révisée, fut officiellement promulguée par le roi Christophe de Bavière. Les deux formes de la loi commune, la première, souvent appelée Loi de Magnus Eriksson, et la seconde, appelée Loi de Christophe de Bavière, furent utilisées au-delà du Moyen Âge : la version promulguée ne devint l'unique loi suédoise qu'à l'occasion de son impression en 1608 et elle resta globalement valable jusqu'en $1734^{22}$.

Les mesures concernant l'empoisonnement et la magie sont généralement décrites dans les codes (balker) de la loi où sont évoqués les délits les plus graves et divers cas de violences. Dans l'Ancienne Loi du Västergötland, l'article concernant les poisons est placé dans un chapitre nommé prettce or bardaghoe, "Ceci est [le code] des coups", qui rassemble des articles traitant principalement des coups et des blessures. De même, dans la Loi d'östergötland, l'article (XXXI § 1) est placé dans un chapitre ne portant pas le nom de balker, mais intitulé Uapa mal ok sara mal, hor, ran och styld, soit «Causes concernant les violences accidentelles, les blessures, l'adultère, le brigandage et le 
vol $»^{23}$. Dans la Nouvelle Loi du Västergötland, on trouve une allusion dans le Code des causes ne pouvant être rachetées par une simple amende (Orbotce mal balker) (article II, $\S 9$ ), et les dispositions principales sont placées dans le Code de la paix publique (XII) et dans le Code des injustices (X). Dans la Loi d'Uppland, l'article figure dans le code qui rassemble aussi des dispositions variées concernant la rupture de la paix publique qui vont de l'homicide au vol et aux objets trouvés et qui s'appelle Manhwelghis balker. Manhoelghi désigne au sens propre l'intégrité du corps et des biens garantie à tout homme libre. L'article XIX évoque le cas où une femme serait prise en flagrant délit : il prend à la fois en compte le cas où la femme n'a pas causé mort d'homme et le cas contraire. Cet article est repris avec de légères modifications dans le même code des lois du Södermanland (XXXII) et du Västmanland (XVI), mais pas dans la Loi du Hälsingland, dont l'introduction du Manholghis balker annonce un article XXVII « Vm öyce drap ok forgcernigar/De l'homicide sur les îles et par le poison ", qui ne dit finalement rien sur le poison. Dans la Loi du Västmanland, une disposition spécifique insérée dans le Code du thing (Pingmala balker) évoque l'empoisonnement du mari par sa femme. Dans la Loi de Dalécarlie, on trouve seulement dans l'article XI du Code de l'Église la description des châtiments contre la simple pratique de la sorcellerie (trolldom), marquée en particulier par l'usage de cheveux et d'ongles, qui implique des amendes très élevées (jusqu'à 49 marcs $^{24}$ ), voire, si la femme ne peut payer, la lapidation ${ }^{25}$. Il faut noter que, dans la Loi de Gotland, qui date de la première moitié du XIII ${ }^{\mathrm{e}}$ siècle, et dans la Loi urbaine de Visby, dont le plus vieux manuscrit, en allemand, date des années 1340, on ne trouve pas d'article spécifique sur le sujet, mais, nous le verrons, les empoisonneurs n'en sont pas moins voués au bûcher.

11 Dans les lois communes à tout le royaume, les articles concernant le poison sont placés dans le Code des causes graves (Höghmalabalker), aux côtés des dispositions concernant le meurtre caché (morp), le meurtre d'enfant, la bigamie et les crimes de haute trahison. Dans la Loi de Magnus Eriksson, l'empoisonnement (forgärning) est évoqué à deux reprises : l'article $\mathrm{V}$, qui traite le cas où un homme ou une femme tue une personne en utilisant la sorcellerie ou l'empoisonnement et l'article XII, qui reprend, en le complétant légèrement, l'article XIX du Manhoelghis balker de la Loi d'Uppland. La Loi de Christophe de Bavière reprend aussi sans grandes modifications ces deux articles (respectivement les articles VI et XV). Alors que le Bjärköarätten n'évoque que rapidement l'empoisonnement dans son article XXXVI, la Loi urbaine emprunte, dans son Code des causes graves, les mêmes dispositions que celles de la Loi de Magnus Eriksson (articles IV et XI) en les adaptant à la procédure d'un tribunal urbain.

\section{Les peines}

12 Ce n'est donc pas dans les codes spécifiquement réservés à l'homicide ou au meurtre (drapabalker) que se trouvent les dispositions concernant le crime par poison. Ce crime relève d'une législation qui envisage, quelle que soit leur nature, les cas les plus graves. Il est, en effet, assimilé à un morp, c'est-à-dire à la forme la plus horrible de crime ${ }^{26}$, le meurtre caché, s'opposant en cela au drap, un homicide perpétré au vu et au su de tous, par exemple par accident ou sous le coup de la colère ou encore par vengeance ${ }^{27}$. Pour ce type de crimes, la sanction est claire, comme le rappelle la Loi d'Uppland:

a hwar mon warpce sannir fore morpe. giceldin liff sitt fore ${ }^{28}$.

(Toutes les fois que des hommes seront convaincus de meurtre secret (morp), ils le paieront de leur vie.) 
Ce crime peut aussi relever de ce que l'on nomme un nipingsvoerk, une action tellement honteuse qu'elle ruine l'honneur, la fama, de celui qui l'accomplit ${ }^{29}$. Ainsi, dans la Nouvelle loi du Västergötland, il est précisé dans le Code des causes ne pouvant être rachetées par une simple amende : «Det œer nißings væerk at for gioræe kono celler manni ${ }^{30}$ / c'est un crime honteux que d'empoisonner un homme ou une femme». Notre traduction par « empoisonner " ne reflète que très partiellement le sens de for giorce, dans la mesure où l'usage du poison relève d'une catégorie plus large, celle des crimes liés à la magie.

Ce sont les liens avec la magie qui expliquent la sévérité des lois suédoises. Tenter de distinguer radicalement crime par poison et sorcellerie, comme l'a fait Ragnar Hemmer semble anachronique. Comme le rappelle Bengt Ankarloo, on considérait que la préparation des poisons exigeait des connaissances occultes ${ }^{31}$. Le veneficium a toujours partie liée avec le maleficium, même lorsque le mot sorcellerie (trolldom) n'apparaît pas de façon explicite comme dans la Loi d'Uppland et les lois du Svealand qui s'en sont inspirées. Le fait que l'on trouve dans l'article $\mathrm{V}$ de la Loi de Magnus Eriksson l'expression "forgör mep trulldom celloe andrum forgerningum/tue en usant de sorcellerie ou d'autres pratiques magiques » indique, que tout en formant une seule catégorie, ce crime peut apparaître sous des aspects variés, la sorcellerie proprement dite en étant la manifestation la plus spectaculaire. Le poison relève d'un savoir secret, de la magie, qu'il s'agisse d'une substance réellement toxique ou d'une potion faite à partir "d'ongles et de cheveux». Même en médecine, l'ingestion d'une potion peut s'accompagner d'incantations qui en augmentent l'efficacité et une action à distance peut être considérée comme tout aussi toxique qu'un poison ${ }^{32}$. Les législateurs ne tranchent donc pas face aux multiples facettes de ce crime: dans l'article XIX du Manhoelghis balker de la Loi d'Uppland, et dans tous les articles qui s'en inspirent, ils retiennent à la fois l'usage de ces pratiques illicites, punies même si elles ne conduisent pas à la mort de la victime, et l'homicide commis par le recours à ces pratiques :

Si une femme utilise du poison/un maléfice (bøer forgiceningæer) contre un homme et qu'elle est prise sur le fait, on doit alors la saisir et l'enchainer, puis la conduire au thing en prenant avec elle le poison (forgicorningæer). Douze hommes doivent établir si elle a commis le maléfice (forgicerningæer) ou non et si elle a été prise sur le fait ou non. S'ils l'acquittent, elle est innocente. S'ils la condamnent, elle doit être soumise à une amende de 40 marcs. Cette amende doit être divisée en trois : le roi en prend une part, l'accusateur, la deuxième et la centaine (hundare $e^{33}$ ), la troisième. Si quelqu'un en est mort et qu'il y a procès, douze hommes doivent établir le fait. S'ils l'acquittent, elle est innocente. S'ils la condamnent, elle doit être brûlée et son héritier prend ses biens. Si l'accusateur lui octroie la vie, elle devra s'acquitter d'une amende de 100 et de 40 marcs, qui sera partagée comme les autres amendes de 100 marcs. Si elle est aussi condamnée pour maléfice (forgiorningum), le paysan (bonde ${ }^{34}$ ) qui l'a enchaînée est innocent. ${ }^{35}$

Le châtiment lié à l'usage de poisons ou de pratiques magiques est très sévère: 40 marcs constituent une somme énorme. S'il y a mort d'homme, la sévérité de la peine s'explique par le fait que tout meurtre secret (morb) doit être expié - fait relativement rare dans le droit suédois - par la mort, comme le rappelle le troisième alinéa du même article. Mais la forme du châtiment montre qu'il s'agit d'un crime considéré comme tout à fait spécifique. C'est normalement la lapidation qui doit punir une femme reconnue coupable de crime ${ }^{36}$. Le bûcher n'est évoqué que dans un seul autre article de la Loi d'Uppland, comme punition de l'incendie criminel d'une ferme, si le propriétaire meurt dans le feu ${ }^{37}$. De plus, il s'agit là d'un châtiment nouveau dans le droit suédois ${ }^{38}$, 
probablement introduit sous l'influence de l'Église dont des membres éminents, comme le chanoine Andreas And, avaient participé à la rédaction de la loi.

Cette spécificité du châtiment se retrouve également dans l'article XXXVI du Bjärköarätten :

Si un homme en tue un autre et qu'il est convaincu par six hommes, il subira le supplice de la roue ou il doit se défendre avec le serment de douze hommes. Le même droit s'applique pour l'incendiaire criminel. Si une femme assassine quelqu'un, elle sera enterrée vive. $\$ 1$ Celui qui donne du poison (forgauor) à un autre, qu'il s'agisse d'un homme ou d'une femme, sera brûlé sur le bûcher. ${ }^{39}$

Dans toutes les autres lois suédoises, le crime de poison et les pratiques magiques auxquelles il est lié sont aussi très durement châtiés. Ce sont, en effet, les peines les plus lourdes qui s'appliquent à chaque fois: la proscription, pour les lois du Västergötland et la peine de mort, dans les autres lois. Il faut noter que la proscription n'est qu'une des formes de la peine de mort, puisque le coupable est offert à la vengeance de la famille de la victime. Ainsi, l'article VIII du Code des coups de l'Ancienne loi du Västergötland précise :

Si une femme empoisonne (firigcr) un homme et si tout le jury (næemd) de la centaine (härad) la déclare coupable, elle doit être laissée en paix un jour et une nuit dans la forêt. Puis on doit la déclarer (dömce) bannie (vgill $\left.{ }^{40}\right)$ pour l'héritier et le plaignant et ensuite la tuer. ${ }^{41}$

Dans la Nouvelle Loi du Västergötland, cet article a été repris dans le Code de la paix publique et il est également précisé à la fin que la femme, bannie, doit être tuée là où on la trouvera ${ }^{42}$. Une autre allusion à ce crime est faite dans l'article $\mathrm{X}$ du Code des injustices : «Si une femme empoisonne (firigcr) une vache ou du bétail, une femme ou un homme, si elle est prise sur le fait, elle perd la vie pour cela ${ }^{43}$ ». La peine ne saurait étonner dans le cas d'un homicide, mais, comme le rappelle Bengt Ankarloo ${ }^{44}$, elle apparaît particulièrement lourde dans le cas où c'est un animal qui est tué, forfait normalement sanctionné par des amendes allant de 27 à 40 marcs. La suite de l'article X est assez confuse, mais il semble, comme le précise Elias Wessén, que le but de l'article est de prendre en compte le cas où il n'y aurait ni prise en flagrant délit, ni mort constatée. Dans ce cas, la femme convaincue de s'être livrée à une tentative d'empoisonnement ou des pratiques magiques devait payer trois fois neuf marcs et l'évêque de Skara devait toucher trois marcs sur la part qui revenait au roi ${ }^{45}$.

La Loi d'Östergötland prévoit la lapidation en cas de flagrant délit, sans même qu'il y ait eu mort d'homme. L'article est particulièrement intéressant, car c'est le seul à évoquer explicitement les traces visibles laissées par le poison :

Si une femme est accusée de sorcellerie et prise sur le fait et que cela est confirmé par des témoins fiables, alors elle a perdu sa vie et on doit la lapider. Maintenant, si on l'accuse et que de véritables signes d'empoisonnement (sanna forgorningar) sont visibles sur le paysan ou sur sa familia ou sur son bétail, elle doit se défendre avec le serment de trois fois douze hommes (brcetylftum epe ${ }^{46}$ ) ou payer une amende de 40 marcs. Si elle est soupçonnée mais qu'il n'y a aucun signe sur le bétail, elle doit se défendre avec le serment de douze hommes ou payer une amende de trois marcs. ${ }^{47}$

Dans cette loi, ce n'est pas le crime en lui-même qui est condamné, mais l'usage de poisons ou de maléfices pour lequel les sanctions sont donc modulées entre le flagrant délit et l'absence de toute trace.

21 Dans les lois du Svealand, c'est le bûcher qui punit l'empoisonneuse. En cas de flagrant délit, les lois nationales et la Loi urbaine, qui s'inspirent directement de l'article XIX du 
Manhoelghis balker de la Loi d'Uppland, prévoient aussi la mort sur le bûcher pour le ou la coupable. En revanche, contrairement à ce que stipulait la Loi d'Uppland, il n'y a plus de rachat possible et l'appartenance de ce crime, et des autres crimes décrits dans le même code, aux Orbotce mal est clairement affirmée ${ }^{48}$.

S'il n'y a pas flagrant délit, le Code des causes graves de la Loi de Magnus Eriksson prévoit dans son article V: «man skal stceghla ok kunu sten $a^{49} /$ un homme subira le supplice de la roue et la femme sera lapidée ». Le même article apparait avec une légère modification dans la Loi urbaine et dans la loi de Christophe de Bavière ${ }^{50}$ : si le coupable est une femme, elle sera brûlée (bræennas) et non lapidée. La lapidation, qui punit la plupart des crimes décrits dans le code des causes graves de la première loi nationale, est remplacée systématiquement par le bûcher dans celle de 1442. Si la peine perd de sa spécificité, elle demeure une des plus graves inscrites dans le droit suédois.

L'usage du poison, quels que soient son mode opératoire et son résultat, appartient à la catégorie des crimes les plus graves. Un autre signe de cette spécificité est la législation concernant la parole. Il existe deux niveaux d'invective qui sont punis par les lois du Västergötland: l'insulte, désignée par le terme ukvœepins ord, et l'injure grave, dite firnarorb, qui blesse gravement l'honneur de la personne, en raison, par exemple, de ses allusions à l'inceste ou à la bestialités1. L'accusation, spécifique aux femmes, d'être une sorcière et de pouvoir empoisonner les hommes et les animaux relève seulement de la première catégorie. Ainsi, dans l'Ancienne Loi du Västergötland, on trouve dans le Code des injustices (en réalité un chapitre formés d'articles très hétéroclites) une petite liste de ces insultes (article V § 1 ) :

«Je t'ai vue chevaucher la barrière de l'enclos, les cheveux défaits, sous l'apparence

d'une sorcière, au moment où il faisant autant jour que nuit. » Si on dit qu'elle peut empoisonner les femmes et les vaches, c'est une insulte. ${ }^{52}$

Dans la Nouvelle Loi du Västergötland, ces deux éléments ont été séparés l'un de l'autre ${ }^{53}$. Faut-il, comme Elias Wessén, penser que ce passage a été déplacé et qu'il est intrinsèquement lié à la première insulte, comme dans la version antérieure de la loi ${ }^{54}$ ? Étant donné la nature du poison, ses liens avec la sorcellerie ne peuvent être mis en doute; cependant, le fait que les deux remarques soient séparées signifie sans doute aussi que l'on pouvait distinguer entre les éléments les plus pittoresques de l'imaginaire maléfique et l'usage des poisons, entre l'accusation de sorcière et celle d'« empoisonneuse ", ou de personne capable de faire mourir par ses maléfices. Ainsi, dans le Bjärköarätten (article XXI), parmi les insultes faites à une femme - insultes qui peuvent conduire à une amende de six marcs -, on trouve "trolkono celler fordapu/ sorcière ou empoisonneuse ». Dans le Code de l'hôtel de ville (Radzstuffw balker) de la Loi urbaine (article XXXI), figurent parmi les insultes retenues forwnna trulkonu, «sorcière dévoyée " et forwnna fordcedho, "empoisonneuse dévoyée». Comme les autres, ces insultes proférées devant témoins donnent lieu à une amende de 12 marcs. Si le fautif ne peut payer, il est condamné au pilori, au bannissement et, en cas de retour dans la ville, il peut avoir la langue arrachée ${ }^{55}$.

Une des rares allusions à un empoisonnement que l'on trouve dans un document de la pratique à l'époque médiévale relève de ces fausses accusations, liées à une dispute autour d'un héritage. L'affaire est présentée dans une lettre de Kalmar du 25 juin 1417 : dix témoins reconnaissent avoir entendu Bryniolf Magnusson dénoncer Sven Lax au sujet d'accusations que celui-ci aurait proférées sur Birgitta, la fille de Bryniolf, qui était aussi sa propre belle-fille. La veuve, Birgitta, aurait selon lui empoisonné (schulde 
forgiffwidh haffwa) son mari Lasse Svensson. Sven nia l'accusation, qui ne put être étayée par aucune preuve ${ }^{56}$, mais l'affaire fut prise au sérieux et elle mobilisa dix témoins. Elle n'eut pas de suites connues et quelques jours plus tard, le 29 juin, Sven Lax ainsi que son gendre et une personne nommée Störia Djeken adressaient à Bryniolf Magnusson une lettre où ils reconnaissaient avoir procédé au partage de l'héritage entre Birgitta et eux $^{57}$.

L'usage des poisons et des maléfices, qui relevait des crimes les plus graves, devait toutefois être exceptionnel à en juger par la rareté des articles dans les lois, pourtant prolixes dès qu'il s'agit de décrire les circonstances et les acteurs des autres types d'homicide. Les protagonistes, empoisonneurs et victimes, et les circonstances sont cependant évoqués avec suffisamment de précisions pour que puissent se dessiner l'imaginaire et les usages du crimen veneficii.

\section{Acteurs et contextes de l'empoisonnement}

Plusieurs exemples cités l'ont déjà montré : le législateur suédois, dès le XIII ${ }^{\mathrm{e}}$ siècle, fait de la sorcellerie et de l'usage du poison une spécialité féminine. Les lois du Götaland comme les lois du Svealand considèrent presque exclusivement l'empoisonnement (forgärning) comme un crime féminin ${ }^{58}$. De même, dans les articles qui donnent des listes d'insultes caractéristiques pour les hommes et les femmes, on ne trouve pas d'équivalent masculin pour « sorcière » ou " empoisonneuse ». Pourtant, la femme est considérée comme une mineure par la législation suédoise. Elle ne participe pas au thing, ne peut témoigner en justice et, selon la situation de la femme, le père, le mari ou le tuteur est considéré comme pénalement responsable de ses actes et c'est lui qui doit être châtié à sa place. Toutefois l'usage de pratiques magiques constitue une exception notable ${ }^{59}$ comme le rappelle, par exemple, le Code du vol (article $5 \$ 2$ ) de l'Ancienne Loi du Västergötland :

konce cer ovormaghi hvn a eigh hug ok eigh hangce utcen firi trolskap. ${ }^{60}$

(Comme la femme est mineure, elle ne peut être égorgée ou pendue, sauf en cas de sorcellerie).

Faut-il considérer que ces articles ne font qu'enregistrer des croyances largement répandues en Suède sur l'existence de sorcières plutôt que de sorciers ${ }^{61}$ ? Il apparaît que cette féminisation de la sorcellerie et de l'empoisonnement n'est pas une spécificité suédoise ${ }^{62}$. Même si l'absence de données factuelles empêche a priori d'y voir autre chose qu'une expression de misogynie, les lois ne sauraient être considérées comme le simple reflet de la société et des pratiques réelles : elles tentent de maintenir la paix publique et de définir pour cela la place de chacun dans la société. Elles proposent donc des normes comportementales. Dans les lois des provinces, c'est le point de vue du bonde qui prévaut : il y apparait en père ou en mari et il exprime dans la loi sa position dominante vis-à-vis de sa femme et de sa fille. La définition de ces dernières comme mineures ne les exclut pas de la loi, surtout lorsqu'il s'agit d'exprimer les propres craintes du bonde face à leurs éventuelles déviances. La législation sur les poisons constitue donc une loi d'exception. Tout se passe comme si poisons et maléfices permettaient à la femme d'échapper à la sphère traditionnelle contrôlée par l'homme : mais en menaçant les fondements mêmes de cette sphère et en échappant à la surveillance à laquelle elle doit normalement se soumettre, la femme se trouve désormais confrontée directement à la loi. Les articles sur les poisons et les maléfices 
doivent donc permettre, en dernière instance, de colmater les brèches - forcément magiques - apparues dans le contrôle exercé par le chef de famille.

Les articles qui envisagent la femme en empoisonneuse peuvent, en effet, s'inscrire dans le cadre familial. Ainsi, l'articleXXI du Code du thing de la Loi du Västmanland met en scène le mari, sa femme et son héritier :

Un paysan (bonde) s'adresse à sa femme et dit: Tu m'as empoisonné! ( $p u$ hafwcer forgiort mic). Si le paysan meurt de cette maladie (sot) et si son héritier porte la même accusation que celle portée par le paysan, elle doit alors prouver qu'elle ne l'a pas fait avec trois témoins et le serment de trois fois douze hommes. Si elle échoue à fournir les serments, qu'il en soit comme pour les autres meurtres (morp). (...) Personne n'a le droit d'accuser la femme dans une telle affaire si ce n'est le paysan et son héritier légitime. ${ }^{63}$

De même, l'articleXV du Code de l'héritage de l'Ancienne Loi du Västergötland évoque le cas où une femme tuerait l'enfant que son mari aurait eu d'un précédent mariage :

Si une femme empoisonne ${ }^{64}$ (firiger) son beau-fils (ou sa belle-fille) et si elle veut céder l'héritage à ses propres enfants, elle doit se défendre avec le jury (næmd) de la centaine (härad). Si elle n'arrive pas à montrer son innocence, elle a alors perdu sa vie et sa sécurité (fred). ${ }^{65}$

La volonté de capter l'héritage au profit de ses seuls enfants apparait comme une circonstance propice au déploiement des ruses féminines dans le cadre familial. Audelà du foyer, c'est tout le domaine du paysan-propriétaire qui peut se trouver menacé par les venins féminins. Ainsi, l'article XXXI du Vådamålsbalk de la Loi d'Östergötland, évoque le cas où une femme empoisonnerait, sans les tuer, le paysan, les habitants de sa ferme ou son bétail ${ }^{66}$.

En revanche, toutes ces spécificités s'effacent dans les lois urbaines et les lois nationales. L'article XXXVI du Bjärköarätten envisage le cas où le poison serait donné par une homme ou par une femme. Il prévoit le même type de châtiment dans les deux cas. La Loi urbaine et les deux lois nationales, alors même qu'elles reprennent les articles de lois antérieures, introduisent toutes la figure nouvelle de l'empoisonneur. Ainsi, dans l'article $\mathrm{V}$ du Code des causes graves de la Loi de Magnus Eriksson, il est précisé :

Si un homme empoisonne un homme ou une femme et une femme, une femme ou un homme, si bien qu'il ou elle en meurt, qu'il ou elle perde la vie pour une telle action. ${ }^{67}$

L'article XII du même code, qui reprend l'article XIX du Manhoelghis balker de la Loi d'Uppland,stipule :

Si une femme ou un homme utilise du poison (brer forgiceningcer) contre un homme et qu'elle est prise sur le fait, on doit alors la saisir et l'enchaîner, puis la conduire au thing en prenant avec elle le poison (forgicerningcer).

Le texte utilise seulement les pronoms féminins et il faut attendre la Loi de Christophe de Bavière pour que le passage soit entièrement récrit avec l'alternative «il ou elle ». Cependant, l'article équivalent de la Loi urbaine comporte l'alternative tout au long du texte :

Si un homme ou une femme use de poison et est pris sur le fait, on doit la ou le prendre avec le poison et l'amener au tribunal de la ville. ${ }^{68}$

De même, dans la Loi urbaine et dans les lois nationales, l'article sur la femme tuant son beau-fils a été modifié et stipule désormais que le meurtrier peut être " man eller kona ${ }^{69}$ / un homme ou une femme». 

d'un type particulier de crime. Il est peu probable, comme le montre la persistance de l'insulte "empoisonneuse » dans la Loi urbaine, que cette transformation révèle un changement des mentalités ${ }^{70}$. C'est plutôt la manière dont l'institution pense désormais le crime qui change ${ }^{71}$. Une hypothèse serait, en effet, que, dans les lois urbaines et les lois nationales, le point de vue adopté par la loi a changé : il n'est plus seulement celui du paysan (bonde) et le foyer et la ferme ne sont plus les seuls lieux où s'envisage le crime par poison ou par maléfices. Un point de vue supérieur, englobant, celui de la ville et de son conseil d'un côté, celui du pouvoir royal de l'autre, s'impose à tous. La perspective du bonde, pour lequel les crimes par poison ou maléfices ne sont pas des crimes masculins, s'efface devant l'intérêt supérieur d'une institution qui ne peut, au risque de penser le crime de manière incomplète, exclure l'homme, le bonde lui-même, des coupables potentiels.

C'est sans doute pour la même raison, mais sous l'influence de l'Église, que l'on trouve dans la Loi de Christophe de Bavière, un article dans lequel le criminel et la victime se confondent. En effet, l'article IV du Code des causes graves évoque pour la première fois dans une loi suédoise, le suicide. Ce qui semble particulièrement intéressant au sujet de cet article c'est le fait que le législateur élargit encore sa perception pour envisager non plus seulement un individu face aux autres, mais, grâce à une mise en perspective plus englobante, un individu face à lui-même :

S'il se produisait malheureusement qu'une personne (menniskia) se tue elle-même (forgör sik siellff), quelle que soit la manière dont cela puisse se produire, elle sera conduite dans la forêt et sera brûlée sur un bûcher. ${ }^{72}$

Malgré l'usage du verbe forgör, il serait sans doute abusif de réduire le contenu de cet article au seul cas du suicide par poison. L'insistance sur la variété des moyens utilisés donne à ce verbe un sens très général, qui inclut toutefois l'usage du poison.

Il n'était pas rare, comme le rappelle Alexander Murray, que les corps des suicidés subissent les mêmes peines que les meurtriers et soient pendus ou brûlés, ce dernier cas étant attesté dans les lois en France et dans l'Empire ${ }^{73}$. Dans la loi suédoise, seul le corps de celui qui n'avait pas toute sa raison (affwita) échappe au bûcher et a le droit d'être enterré, mais hors du cimetière : il s'agit sans doute d'envisager le cas où une personne consommerait sans le vouloir une substance toxique ou, plus probablement encore celui d'un fou qui se tuerait, même si théoriquement un fou devait, devant témoins, être déclaré tel par sa famille qui était alors obligée de le tenir enfermé ${ }^{74}$. Même si le suicide par poison n'est sans doute pas le seul envisagé dans cet article, cet acte est considéré comme un crime tout aussi grave. Une autre raison explique sans doute le traitement réservé au corps du suicidé : ayant commis en toute conscience un acte abominable, il risque de contaminer, comme un poison, le reste du corps social et le brûler permet d'empêcher cette contagion ${ }^{75}$.

La Loi nationale promulguée en 1442 propose donc, autour du crime par poison, une riche palette de possibilités où hommes et femmes peuvent être considérés comme coupables et victimes. Notons toutefois qu'il existe un cas particulier où même une loi provinciale peut envisager la possibilité pour un homme de commettre un crime par poison contre une femme, cas qui inverse les circonstances par ailleurs décrites dans les lois. Il s'agit d'une disposition qui se trouve à l'article VII du Code de l'héritage (Ärfpabalker) de la Loi d'östergötland. Dans ce même code, qui a pour fonction de régler les successions, le même article fut repris dans la Loi de Magnus Eriksson (article IX), dans 
la Loi urbaine (article VIII) et dans la Loi de Christophe de Bavière (article X). Il envisage le cas où une femme enceinte serait tuée par l'héritier de son mari :

Un paysan (bonde) se marie, habite avec sa femme, a avec elle un enfant, mais ce paysan meurt avant qu'elle ne se sache enceinte. L'héritier du paysan se présente et prend son héritage et dit qu'ils n'avaient pas d'enfant ensemble et c'est pourquoi tu ne dois pas hériter de lui ${ }^{76}$. Elle ne sait pas qu'elle est enceinte et cède l'héritage. On commence à dire qu'elle est enceinte et celui qui a l'héritage apprend qu'elle est enceinte. Alors, il se rend chez elle et la tue (drcepcer), car il répugne à perdre l'héritage, ou il lui prend la vie en l'empoisonnant (møep ogcerningum). Elle est enterrée, mais il est révélé, après qu'elle a été enterrée, qu'il lui a pris la vie. S'il se trouve que les témoins qui l'affirment sont fiables, on doit la sortir de terre et lui ouvrir le corps. Si un enfant ou une semblance d'enfant est trouvé dans son corps, [cet enfant hérite de son père et la mère hérite de l'enfant et son héritier hérite d'elle ${ }^{77}$. Ici, c'est celui qui est mort et non-baptisé qui hérite et celui qui est vivant et chrétien qui cède l'héritage, car personne ne doit tuer pour un héritage ${ }^{78}$.

41 Le but de ce passage n'est pas de prévoir les sanctions contre le meurtrier, mais d'envisager un cas tout à fait exceptionnel où l'héritage passe par un enfant qui n'est pas né et qui n'a donc pas pu être baptisé: or, en théorie, seul un chrétien peut légalement hériter ${ }^{79}$. Cette fois, la perspective n'est plus la même que dans les autres articles évoquant le poison : il ne s'agit pas, comme dans l'article XXI du Code du thing de la Loi du Västmanland, de punir une menace venimeuse émanant du foyer même du bonde ou, comme dans l'article XXXI du Vådamålsbalk de la même loi, une menace pour le bonde, sa familia et son cheptel ${ }^{80}$. L'enjeu est désormais de protéger la femme du bonde et, à travers elle, la vie de ses héritiers. Dans cette perspective, il n'y a donc pas d'obstacle particulier à envisager qu'un homme puisse recourir aux poisons. Il n'est pas exclu que ce cas relève de la jurisprudence, mais il apparaît aussi comme une manière particulièrement intéressante de penser l'usage du poison. Si, dans les circonstances envisagées, il est probable que ce soit un homme qui réclame l'héritage, il est aussi plus probable qu'il ait recours à un moyen discret de supprimer sa victime. En tuant la femme pour tenter de dissimuler l'existence d'un héritier, il permet la sauvegarde de l'héritage du côté des agnats. Mais un homicide (drap) l'exposerait à des sanctions directes. Le législateur a donc envisagé le cas où l'héritier aurait recours au poison, c'est-à-dire à la forme la plus secrète du meurtre, celle qui ne met pas en évidence les intentions du criminel. C'est la raison pour laquelle la motivation doit être recherchée, même si la victime a déjà été enterrée. Si l'on déterre la femme, ce n'est donc pas pour retrouver d'éventuelles traces de l'empoisonnement - généralement les seules preuves présentées devant un tribunal sont les poisons eux-mêmes ${ }^{81}$ - mais pour constater ou non la présence du fœtus et déterminer ainsi la succession.

Un des très rares cas connus de condamnation au bûcher pour empoisonnement s'éloigne considérablement des circonstances envisagées par les lois. Il se déroule à Gotland, dans la ville de Visby, dont la législation ne comporte pas de passage spécifique sur le poison. Bien qu'elle fût soumise à l'impôt royal et rattachée au diocèse de Linköping, l'île bénéficiait d'une large autonomie au sein du royaume de Suède et sa ville principale, Visby, était une cité puissante, fondatrice, avec Lübeck, de la Hanse ${ }^{82}$. C'est dans ce cadre que les bourgmestres et les conseillers de Visby envoient à leurs homologues de Rostock une lettre, dont la copie a été conservée dans les registres de cette ville. Dans ce document, qui fut sans doute écrit en mai ou en juin 1350, au moment où la Suède et Gotland étaient touchées par la Peste noire ${ }^{83}$, ils leur annoncent avoir condamné neuf empoisonneurs et leur font part des révélations faites par deux 
d'entre eux au moment de mourir sur le bûcher. Comme ailleurs, le contexte de la peste est favorable aux rumeurs de complots.

Le premier est décrit comme un organiste (organista). Sur le bûcher, au moment de mourir, il proclame devant le peuple réuni qu'il «a empoisonné de ses venins tous les puits dans les villes de Stockholm, de Västerås et d'Arboga ainsi que des étangs, des étendues d'eau et d'autres puits sur son passage lorsqu'il a traversé la Suède ${ }^{84}$. » Les autorités de la ville assurent avoir retrouvé le poison et l'avoir détruit. Il ajouta :

Pulverem quendam coxerat et temperaverat, de quo unus hominum in tota terra Godlandie, si vixisset ad unius anni circulum, vivus non debuit remansisse, suis duntaxat exceptis. ${ }^{85}$

Après la Suède continentale, c'est donc toute l'île de Gotland qui s'est trouvée menacée par les poudres empoisonnées de cet homme qui avoue ne pas agir isolément. C'est un véritable complot qui se trouve dévoilé (plurimi essent de sua societate): de riches marchands et des notables, saisis de folie, font partie de cette société qui est répandue dans le monde entier. Ils sont reconnaissables au fait qu'ils sont marqués d'un signe grec ou hébreu. La troisième révélation permet de retrouver des accusations plus classiques : "Je ne sais que vous dire de plus, mais toute la Chrétienté est empoisonnée par les juifs et les pires d'entre nous ${ }^{86} »$. Si ailleurs, ces fausses rumeurs, qui accompagnent, voire précèdent l'arrivée de la peste, sont liées à la présence de juifs parmi les chrétiens, elles ne touchent à Visby que l'imaginaire dans la mesure où on n'y trouve pas d'habitants juifs et les juifs y sont seulement connus par les textes ${ }^{87}$. Cependant, les clercs sont aussi touchés par la rumeur. Parmi les neuf accusés figuraient aussi deux hommes qui se faisaient passer pour clercs. L'un des deux prit la parole sur le bûcher pour annoncer que toute la Chrétienté était perdue (tota Christianitas perdita est) et que tous devaient se méfier des prêtres et des religieux. Il ajoute que

le lundi de Pentecôte, lorsqu'il célébrait la messe dans l'église Saint-Olav avec nous, il avait empoisonné de venin le manipule qu'il avait pour faire cet office, de telle sorte que tous ceux qui l'avaient embrassé lors de l'offertoire étaient morts trois ou quatre jours plus tard et tous ceux qui étaient venus les visiter de même. ${ }^{88}$

Saint-Olav était une église paroissiale située au nord de la ville, dans un quartier d'artisans et de petites gens que fréquentaient peu les grands marchands ${ }^{89}$. Contrairement à ce qu'écrivent les conseillers, ils n'ont sans aucun doute pas euxmêmes assisté à cette messe. Et l'affirmation selon laquelle trois prêtres et plusieurs fidèles de la paroisse sont effectivement morts peu après doit être évidemment liée à l'épidémie de peste qui touchait alors la ville et qui frappait sans doute plus durement ces quartiers populaires.

L'affaire des poisons de Visby permet de constater que Gotland n'était pas isolée en Occident: l'atmosphère paranoïaque fit ressortir des croyances et des peurs qui relevaient d'une culture commune malgré l'originalité du manipule empoisonné. Cette atmosphère dura quelque temps, sans doute celui de la maladie, puisqu'un homme du nom de Tidericus, accusé d'avoir empoisonné des puits dans plusieurs villes d'Allemagne du nord, fut également arrêté à Visby. Il affirma avoir reçu ses poisons de deux juifs nommés Aaron et Moïse. Il fut brûlé le $1^{\mathrm{er}}$ juillet $1350^{90}$, peu de temps après l'exécution des neuf premières victimes de la rumeur.

47 Les autres affaires d'empoisonnement suédoises n'ont pas beaucoup plus de consistance. Elles s'inscrivent dans des contextes politiques qui constituent généralement le principal ingrédient des venins que les chroniques mettent en scène. 


\section{Le poison politique} seront présentés ici relèvent de la fausse rumeur et doivent être interprétés comme des tentatives de manipulation. Le cas du roi Inge, qui sera présenté en premier, est peutêtre une exception : son empoisonnement reste impossible à démontrer, mais, s'il a été inventé, les raisons nous échappent tout autant. Les chroniques rimées présentent des accusations plus grossières. Pourtant, il arrive que ces fictions se répandent. Ainsi, malgré son apparition dans un pamphlet qui cherchait manifestement à salir le couple royal, mais qui s'inspirait des révélations de sainte Brigitte, le portrait de la reine Blanche en empoisonneuse a connu un long succès.

\section{Vreta, lieu venimeux}

Le seul empoisonnement d'un roi suédois dont l'historiographie garde la trace est celui d'Inge le Jeune. Dans la liste des rois chrétiens contenue dans le manuscrit de l'Ancienne Loi du Västergötland, on trouve la notice suivante :

Le neuvième [roi chrétien] fut le roi Inge, frère du roi Philippe. Il fut empoisonné avec une mauvaise boisson en Östergötland et il en mourut. La Suède alla toujours bien lorsque ses parents gouvernaient. ${ }^{91}$

Inge le Jeune régna à partir de 1110, avec son frère Philippe, qui mourut en 1118 et le laissa seul au pouvoir. Il est sans doute mort vers $1125^{92}$. Son règne est très mal connu et c'est sur la seule foi de cette notice qu'Inge peut être considéré comme l'unique roi suédois empoisonné. Son successeur, Ragnvald Knaphövde fut tué par les habitants du Västergötland car il avait refusé de donner des otages au moment où il traversait la province pour aller au thing faire reconnaître son élection ${ }^{93}$. Sa mort prend place dans un contexte qui est bien explicité et qui est justifié par le refus du roi de se soumettre à la loi. Rien de tel pour le roi Inge le Jeune, décrit comme un bon roi, mais pour la mort duquel aucune explication n'est donnée.

51 La liste des rois du manuscrit de la Loi du Västergötland fut diffusée, y compris en latin. Une liste des rois, placée dans le Registrum Uppsalense datant de 1344, apporte toutefois un élément nouveau :

En neuvième lieu, le frère de Philippe, Inge le Jeune, qui tirait son nom du précédent Inge, son oncle, reçut le royaume et, quelque temps après, il mourut empoisonné à Vreta en Östergötland.9 ${ }^{94}$

Situé au nord de l'Östergötland, Vreta était un ancien domaine royal sur lequel fut érigée une église dès le $\mathrm{XI}^{\mathrm{e}}$ siècle. Il est probable qu'avant même la fondation d'un monastère de moniales cisterciennes, il y ait eu à Vreta un monastère de moniales bénédictines, le premier monastère suédois, fondé par Inge lui-même ou par son oncle Inge l'Ancien. La découverte de tombes dans l'église fait de Vreta le lieu possible d'inhumation des rois de cette dynastie, mais rien ne permet d'affirmer avec certitude qu'Inge le Jeune y soit inhumé ${ }^{95}$. L'auteur de la liste aurait-il confondu le lieu de sa mort et son lieu d'inhumation? Dans une version $d u \mathrm{Xv}^{\mathrm{e}}$ siècle, très proche de cette liste, c'est une autre lecture qui est proposée : Postmodum in osgocia veneno impocionatus decessit ${ }^{96}$. Wretis serait donc peut-être une mauvaise lecture de veneno ce qui correspond mieux à une traduction latine de l'expression suédoise «mœed ondom dryk». La présence 
de la tombe d'Inge à Vreta aurait peut-être facilité cette confusion. Comme il s'agit du seul détail donné sur les circonstances de l'empoisonnement, on comprend le succès de cette lecture dans les chroniques à la fin du Moyen Âge.

Dans la Chronique en prose, rédigée sous le premier règne de Karl Knutsson (1448-1457), on trouve une simple paraphrase de la notice et dans la Petite Chronique rimée, où chaque roi s'exprime à la première personne, Inge affirme avoir été empoisonné « $j$ vrätha closter ${ }^{97}$ ", c'est-à-dire dans le monastère même. Vers 1470, dans sa Chronica regni Gothorum, Ericus Olai souligne que le roi fut empoisonné à Vreta. Il transforme la remarque sur le fait que lui et ses parents furent de bons rois en une évocation de la douleur ressentie alors dans tout le royaume et une plainte sur la disparition du sang royal : Sic amore dominandi lucerna in Israel est extincta, sic sanguis regius exterminatus a regn $0^{98}$. L'empoisonnement d'Inge trouve sa place dans les desseins de la Providence, puisqu'il permet l'avènement de saint Éric dont il est dit, sur la foi d'une généalogie de la première moitié du xIve siècle, qu'il avait épousé la fille d'Inge.

Olaus Petri, qui rédigea vers 1540 sa chronique suédoise, est le premier à avancer des explications - anachroniques - pour l'empoisonnement du roi :

Et le roi Inge fut empoisonné à Vreta en Östergötland, par ceux, sans doute, qui ne pouvaient faire autant de mal qu'ils le souhaitaient, car le roi avait l'habitude de châtier ceux qui commettaient des injustices, et il est possible aussi que certains, membres du Conseil, aient voulu ainsi accéder au pouvoir. ${ }^{99}$

Olaus Petri reconnaît toutefois que toutes ses sources ne s'accordent pas sur la mort du roi Inge :

La chronique danoise dit aussi (...) qu'un cheval piétina ce roi Inge, qui pendait avec

le pied pris dans l'étrier, et qui en mourut. Mais nos chroniques suédoises disent unanimement qu'il fut empoisonné à Vreta. ${ }^{100}$

Olaus Petri fait référence aux Gesta Danorum. En effet, Saxo Grammaticus décrit dans le premier chapitre du livre XIII, la mort d'un Inge, piétiné par un cheval fougueux : il ne s'agit pas du roi Inge le Jeune, mais du fils du roi de Norvège, Magnus Berrføtt. Johannes Magnus reprit lui aussi le motif de l'empoisonnement à Vreta, qualifiant simplement au passage ceux qui avaient tué le roi de perditissimis hominibus ${ }^{101}$. L'horreur d'un crime mal connu était capable d'émouvoir quatre siècles après. Quelques années plus tard, le fils de Gustave Vasa, Jean III, faisait ériger à Vreta une tombe pour le roi Inge ${ }^{102}$.

\section{L'accusation d'empoisonnement dans les chroniques rimées}

L'Erikskrönika, qui décrit un temps, les années 1220-1320, bien postérieur au règne d'Inge, ne mentionne le poison qu'une seule fois, dans le cadre de la lutte qui oppose Birger Jarl et le junker Karl. Birger défend les droits de son fils Valdemar au trône et Karl, dont le titre de junker indique qu'il est de famille royale, tente de revendiquer la couronne à son profit :

Junker Karl se réconcilia alors avec lui car il vit qu'il était le plus fort.

Chacun nourrissait de la haine envers l'autre, plein d'animosité et de malveillance, et chacun craignait pour lui-même et s'attendait à une ruse de l'autre.

Le jarl voulut alors le faire empoisonner (forgiffua) :

il n'était pas bien aise de le savoir en vie. 
Un chevalier l'en avertit

et lui conseilla de partir

là où il pourrait vivre en paix

et en sûreté pour sa vie. ${ }^{103}$ l'Ancien était régent du royaume (1470-1497). Elle décrit le règne du roi Christian I ${ }^{\text {er }}$ (1457-1464), la fin du règne de Karl Knutsson et la situation du royaume sous Sten Sture, au moment où la Suède avait quitté l'Union de Kalmar et se trouvait sans roi. La reine Dorothée, «the wärsta qvinna som man kan fynna ${ }^{106} /$ la pire femme que l'on puisse trouver ", est accusée d'avoir voulu empoisonner son fils :

Tout le monde pourrait l'appeler Jézabel.

Il serait juste qu'elle ne vive pas plus longtemps !

Elle voulait empoisonner son fils, le junker Hans

avec une poule qui avait été bien rôtie.

Elle le fit sans raison.

Dieu lui donna de la présence d'esprit

afin qu'il en réchappe.

Aujourd'hui, les choses ne se sont pas améliorées :

si elle ne change pas de sentiment,

et si elle arrive elle-même au pouvoir,

elle sera une menace pour la Suède. ${ }^{107}$

61 Les raisons de cette fausse accusation ne sont pas difficiles à établir : ces vers figurent au tout début de la deuxième partie de la chronique, rédigée, à partir de 1487, par un 
auteur favorable à Sten Sture ${ }^{108}$. L'auteur vient de rappeler la mort du roi Christian et en profite pour dresser le portrait de la reine Dorothée. Sa haine pour la reine s'explique aussi par le conflit qui opposait depuis plusieurs années Dorothée au régent au sujet de son douaire (morgongåva), composé du château d'örebro, du Värmland et de Närke. Sten Sture s'en était emparé et avait redistribué leurs revenus à ses fidèles ${ }^{109}$. Lors d'un pèlerinage à Rome en 1475, la reine avait obtenu une bulle du pape assignant Sten Sture en justice et une autre autorisant les archevêques de Lund, de Magdebourg et de Raguse à excommunier ceux qui tenaient les domaines relevant de son douaire. Le procès ne commença qu'en 1479: Sten Sture fut excommunié sans que cela ait le moindre effet et le procès s'enlisa. Au moment où l'auteur de la chronique rédigeait ces vers, la reine s'était à nouveau tournée vers le pape et Sten Sture se trouvait encore une fois menacé d'excommunication ${ }^{110}$.

Le fait que Hans (Jean II), devenu roi à la mort de son père, ait été choisi comme victime s'explique peut-être par le fait que la reine ne ménageait pas ses efforts pour offrir une couronne à son fils cadet, Frédéric ${ }^{111}$, mais aussi, à en croire l'auteur, par son désir de prendre elle-même le pouvoir. Il est certain que la reine travailla activement pour voir se reformer l'Union avec la Suède, en particulier en contactant la papauté et en liant son procès aux négociations entre le Danemark et la Suède, et ses initiatives durent apparaître intempestives. C'est donc contre une femme particulièrement tenace et active sur la scène diplomatique que ces rumeurs sont lancées, sans succès.

La dernière accusation d'empoisonnement concerne à la fois une reine, Christine de Saxe, et son propre médecin. Elle apparaît dans la dernière des chroniques médiévales, la Cronica Swecie, rédigée vers 1520. Cette chronique constituait à la fois une version étoffée de la Petite chronique rimée et une récriture de toutes les chroniques rimées suédoises, complétées jusqu'en 1520. L'originalité de ce texte est de se présenter sous forme de monologues fictifs : chaque roi ou régent y assume en vers la narration de son règne. Dans cette fiction, Sten Sture l'Ancien raconte lui-même la façon dont il est mort. En 1501, Sten Sture avait réussit à reprendre le pouvoir en Suède, mais sa lutte contre le roi danois Jean II fut âpre et il dut assiéger plusieurs places fortes encore occupées par les Danois. La reine Christine, restée à Stockholm, fut faite prisonnière lors de la chute de la ville, mais sa libération fut négociée et en décembre 1503, Sten Sture raccompagna la reine à la frontière danoise : c'est dans la ville de Jönköping, sur le chemin du retour, le 14 décembre 1503, qu'il trouva la mort.

Dans le monologue, Sten rappelle que Christine avait à ses côtés un médecin du nom de Karl qu'elle lui envoie après leur séparation :

Docteur Karl est revenu une fois encore :

je pensais qu'il était mon ami.

Il avait alors quelques affaires

pour lesquelles il avait besoin de mon conseil.

Ensemble, nous avons bu et mangé.

Nous étions tous deux assis devant la boisson et la nourriture :

à ce moment, il m'a empoisonné (J then stwnd monde han mig förgiffua),

car il ne souhaitait pas que je vive plus longtemps.

Lorsque cela a été fait, il est rentré à cheval

chez la reine Christine par le plus court chemin.

J'ai aussitôt chevauché jusqu'à Värnamo.

Je n'avais ni halte ni repos,

plein de douleurs, en sueur et au plus mal.

Je suis arrivé alors à Skillingarud. 
Là, j'ai dû me mettre au lit :

je n'avais plus la force de voyager.

Mes amis ont insisté

pour que j'aille quand même jusqu'à Jönköping.

Le dimanche, je suis arrivé là au plus mal.

Le jeudi suivant, j'étais mort. ${ }^{112}$

L'auteur a rassemblé tous les éléments d'un empoisonnement réussi avec un commanditaire puissant, dont la culpabilité est sous-entendue, la reine, un exécutant zélé et bon connaisseur de potions, le médecin, et une occasion idéale, celle du partage de nourriture. Au moment où l'auteur écrivait ces vers, sans doute après le Bain de Sang de Stockholm, la perfidie danoise se devait d'être à nouveau démontrée. Il n'est sans doute pas à l'origine de la rumeur d'empoisonnement: les circonstances s'y prêtaient et quelques continuations de la Petite Chronique rimée y font aussi rapidement allusion ${ }^{113}$. Mais cette version n'eut pas beaucoup de succès : par exemple, Olaus Petri, qui écrit presque vingt ans plus tard, précise que Sten Sture est mort à Jönköping et ajoute «aff fögifft, som en part mena/par poison, comme quelques-uns le disent ${ }^{114}$ ». La formule montre ses doutes et, surtout, il ne dit rien des coupables.

Dans la Cronica Swecie, la suite du monologue de Sten Sture donne des renseignements moins fantaisistes sur le retour à Stockholm du corps du régent, dissimulé, pour permettre de préparer sa succession dans de bonnes conditions ${ }^{115}$. Un détail peut retenir l'attention: «Mon corps fut déposé dans l'église de la ville, sur l'autel de saint Georges ». En 1489, pour cette église, Sten Sture fit réaliser par Bernt Notke, artiste originaire de Poméranie, une grande statue de saint Georges terrassant le dragon, haute de plus de deux mètres. Cet ensemble, destiné à remercier le saint de la victoire obtenue en 1471 par les Suédois sur les Danois et leurs alliés à Brunkeberg, aux portes de Stockholm, a souvent été interprété comme une représentation de Sten Sture luimême, terrassant le "dragon" danois ${ }^{116}$. Il ne faut peut-être pas chercher plus loin l'origine des venins danois instillés dans les chroniques: Sten Sture avait veillé à indiquer lui-même l'origine de tous les troubles qui affectaient la Suède.

\section{Venins brigittins}

67 Les textes de sainte Brigitte sont eux aussi peuplés de serpents, de dragons et de diables venimeux. C'est dans cet imaginaire toxique qu'il faut situer l'origine de la rumeur dont fut victime la reine Blanche de Namur. Dans un écrit inspiré par la sainte, elle fut accusée d'avoir empoisonné Erik, son propre fils.

Sainte Brigitte utilise fréquemment le poison comme métaphore du mal dans son œuvre. Par exemple, dans une révélation, le chapitre 48 du Liber celestis imperatoris ad reges compilé par Alfonso Pecha, Brigitte évoque le jugement céleste de deux rois, dont l'un est sans doute Magnus Ladulås, mort en 1290. Ce roi apparaît avec ses vêtements royaux et il dévoile sa conscience : il évoque, en particulier, les impôts nouveaux dont il a accablé son royaume, parmi lesquels figure le droit de dépouille. Un diable lui répand alors sur les mains un vase de venin. Puis, on lui apporte un vase rempli d'onguent dont le roi aurait pu se servir pour retirer le poison, mais il n'en fait rien ${ }^{117}$. Le roi Magnus Lådulås était mort depuis longtemps lorsque Brigitte le met en scène, mais la leçon était censée profiter à son petit-fils, Magnus Eriksson. C'est la raison pour laquelle la leçon est tirée : 
En troisième lieu, tu as vu qu'au moment où le roi pensait imposer dans son royaume de nouvelles exactions et des tributs, du venin fut répandu sur ses mains. Cela signifie que les actions du roi étaient dirigées par un esprit diabolique et de mauvaises pensées. Ainsi, comme le venin produit dans le corps une agitation et un refroidissement, le roi a été troublé et agité par des pensées et des avis pernicieux, cherchant les moyens par lesquels il pourrait obtenir les terres et les biens d'autrui et l'or des voyageurs. ${ }^{118}$

Le vase d'onguent représentait l'antidote, le sang du Christ, sur lequel le roi aurait dû méditer afin d'effacer ses mauvais penchants. Brigitte souligne que ce roi a pu régner jusqu'à sa mort, mais les châtiments qui lui sont réservés en enfer en disent long, malgré l'insistance sur la dimension spirituelle des images utilisées, sur l'imaginaire du meurtre politique qui se dévoile dans la révélation: le désir de voir le roi châtié s'accomplit dans le texte, dans cet au-delà de l'action politique, où le roi est écorché, brûlé et transpercé119. Le poison n'est pas absent de cette exécution fantasmée et prend des formes étrangement maternelles, lorsque le diable chargé de punir le roi propose :

Lions-nous par trois cordes. Que la première corde soit au milieu et qu'elle lie ton nombril au mien de sorte que lorsque j'expirerai, tu feras entrer en toi mon venin et lorsque tu expireras, j'attirerai en moi tes entrailles. ${ }^{120}$

70 Brigitte a précédemment pris soin de décrire le nombril plein de venins bouillants et colorés du diable (vmbelicus apertus ebulliens veneno colorato omnibus coloribus venenosis). Mais le venin ne sert pas seulement à châtier les mauvais rois en enfer : il sert aussi à exprimer le mal que commettent les vivants, en particulier le roi Magnus Eriksson, sa femme Blanche et ses mauvais conseillers. Brigitte connaissait bien la reine Blanche. La fille du duc Jean de Namur et de Marie d'Artois avait épousé Magnus Eriksson en 1335 : dès son arrivée en Suède, Brigitte était devenue sa gouvernante (magistra ${ }^{121}$ ), chargée de lui enseigner les mœurs du pays et sans doute sa langue. Au début très proche du couple royal, qui avait accueilli ses premières révélations avec enthousiasme, elle prit peu à peu ses distances et se mit à exprimer des critiques de plus en plus vives, même après 1349 , date de son départ pour Rome.

La révélation 31 du livre VIII fait partie des rares visions de la sainte. Elle se divise en deux parties distinctes. Le texte commence par la description de la vision reçue par Brigitte : elle voit le ciel au-dessus de Stockholm, lieu de résidence privilégié du roi et de sa cour, où une nouvelle forteresse venait d'être édifiée. Elle décrit le ciel obscurci dans lequel s'affrontent les armées des bons et des mauvais anges, puis, le soleil et la lune aident un dragon (draco) à monter jusqu'à eux. La lune disparaît et le soleil noircit tandis que la terre devient la proie des serpents. Le soleil, enfin, tombe dans l'abîme. La seconde partie, écrite onze ans après, c'est-à-dire lorsque Brigitte se trouvait à Rome, donne l'explication de la vision en se contentant toutefois de nommer les protagonistes par leur fonction et non par leur nom. Le soleil, dont le symbolisme royal est fréquent dans les Révélations, représente Magnus Eriksson. La lune représente la reine Blanche et le dragon, Bengt Algotsson, le favori du roi, mais aussi d'après les allusions de Brigitte, de la reine ${ }^{122}$.

72 En 1353, alors que la situation extérieure était inquiétante, le roi choisit de rompre avec le Conseil en imposant son favori Bengt Algotsson, grand aristocrate suédois, mais membre d'une famille sans responsabilité au Conseil ${ }^{123}$. Bengt, dont la carrière fut exceptionnellement rapide, fut nommé duc de Finlande et de Halland, au mépris de la tradition suédoise qui réservait ce titre aux fils de roi. La Révélation prend sa place parmi les nombreuses critiques adressées au roi pour son attachement à un homme que 
l'aristocratie du Conseil considérait comme un manipulateur et un ambitieux, un obstacle à la défense de ses intérêts. Brigitte ne faisait qu'appuyer les membres du Conseil qui exigeaient son renvoi. Face au refus du roi, son propre fils, Erik Magnusson, élu roi de Suède dès le mois de décembre 1344, et une partie de l'aristocratie, soutenue par la majorité des évêques suédois (six sur huit), se révoltèrent dans le sud de la Suède à partir d'octobre $1356^{124}$. Ils obtinrent l'exil de Bengt Algotsson et la division du territoire du royaume entre Magnus et Erik. Mais la mort d'Erik, en 1359, permit au roi, alors allié au roi danois Valdemar Atterdag, de recouvrer, pour un temps, son pouvoir.

Quelques années plus tard, au moment où Magnus Eriksson était retenu prisonnier par le roi Albert de Mecklembourg, entre 1365 et 1371, fut rédigé un pamphlet en latin qui proposait une critique du règne de Magnus largement inspirée par les révélations de Brigitte. Ce pamphlet, connu sous le nom de Libellus de Magno Erici rege (mais introduit sous le titre Qualiter regnauit rex Magnus), est conservé dans un manuscrit qui se trouvait dans la bibliothèque de Vadstena. Son auteur est un clerc, ou éventuellement un laïc lettré, qui n'a pu être identifié1 ${ }^{25}$, mais qui semble avoir eu accès à la production historiographique de Vadstena et avoir été proche, sinon de Brigitte elle-même, du moins des brigittins. Il s'inspire largement des accusations que la sainte a portées contre le roi ${ }^{126}$ et revient sur le soulèvement des années 1356-1359 en le décrivant, non comme un mouvement seulement dirigé contre le duc Bengt Algotsson, mais comme une véritable révolte contre le couple royal. En effet, plus encore que le roi, c'est la reine Blanche qui apparait comme la principale responsable des malheurs du royaume et qui joue un rôle-clef au moment de la révolte de son fils Erik :

La reine, entendant et comprenant que son fils demeurait ferme dans sa décision, alla trouver le très cruel roi de Danemark et lui promit la Scanie s'il redonnait au duc son honneur. Et il semblait à tous absurde et suspect que la reine se mêlât des affaires d'un tel royaume alors que le roi demeurait en paix chez lui. Cependant, le roi de Danemark approuva les paroles de la reine et il entreprit tant de choses ou en fit tant que les fils de nos fils en parleront encore. Mais parce que le fils du roi prospérait en tout lieu, il fut décidé du jour et du lieu où le père, la mère et les conseillers se réuniraient sous le prétexte de faire la paix. Et lorsque tous se furent retirés, la mère donna à son fils une potion (pocionem) qui le fit mourir moins de vingt jours plus tard. Et dès qu'il sentit son corps faiblir, à l'article de la mort, il dit lorsqu'on l'interrogea: «Ceux qui m'ont mis au monde me font aussi quitter ce monde $» .^{127}$

Nul doute que cette phrase rapportée au style direct est pensée comme une sorte de preuve de la culpabilité de Blanche et de Magnus : comme le mari empoisonné de la Loi du Västmanland accuse de vive voix sa femme, Erik apporte lui-même la confirmation de son assassinat. L'accusation de l'auteur ne repose sur aucun fait réel : il est possible qu'il ait lui-même lancé la rumeur, dans un contexte politique propice à toutes les manipulations, pour justifier le ralliement d'une partie de l'aristocratie suédoise au roi Albert de Mecklembourg. Il n'hésite pas, non plus, à reprendre une autre rumeur, celle du traité secret entre Magnus Eriksson et le roi Valdemar Atterdag au sujet de la Scanie. Lorsqu'en 1360, Vademar s'empare de la Scanie, Magnus est accusé d'avoir simplement donné la province en échange de son soutien lors de la révolte de son fils. Sans doute née dans les milieux aristocratiques suédois hostiles au roi, cette rumeur parfaitement infondée est largement diffusée par Brigitte qui, installée à Rome, reste attentive aux évolutions de son pays ${ }^{128}$. Dans le pamphlet, la rumeur est quelque peu transformée puisque c'est Blanche elle-même qui négocie l'abandon de la Scanie. Cette femme, accusée, dans le même texte, d'avoir corrompu le roi et de l'avoir éloigné de ses 
conseillers ${ }^{129}$, n'est pas une simple empoisonneuse : c'est le royaume tout entier qu'elle met en péril. Reine étrangère accusée des pires crimes, Blanche rejoint donc la liste déjà longue des épouses de souverains accusées de corrompre les traditions nationales ${ }^{130}$.

Aussi fantaisiste soit-il, le pamphlet eut un grand succès dans l'historiographie : dans le poème de liaison, rédigé au milieu $\mathrm{du} \mathrm{xv}^{\mathrm{e}}$ siècle pour attacher les chroniques nouvellement rédigées à l'Erikskrönika, l'empoisonnement d'Erik est évoqué et la culpabilité de sa mère est fortement soulignée ${ }^{131}$. Dans son histoire de la Suède, Ericus Olai rédige, pour rapporter l'empoisonnement, une simple paraphrase du pamphlet, sans oublier les paroles finales d'Erik qui fournissent tout le sel de l'épisode ${ }^{132}$. Vers 1540, Olaus Petri, qui rompt pourtant avec la tradition brigittine et propose, de ce fait, une vision plus mesurée du règne de Magnus Eriksson ${ }^{133}$, reprend sans sourciller les données du pamphlet dans sa Chronique suédoise ${ }^{134}$. Johannes Magnus s'empare lui aussi de l'épisode dans son Historia de omnibus Gothorum Sveorumque regibus. Il montre qu'en donnant le poison, la reine a voulu venger la mort de son favori Bengt qu'Erik aurait assassiné de ses propres mains ; cet ajout est faux, puisque Bengt est mort en 1360, mais il colore incontestablement le drame. L'archevêque d'Uppsala donne à Blanche le premier rôle en ne proposant qu'une paraphrase incomplète des paroles, désormais célèbres, prononcées par Erik au moment de mourir : Nam a crudelissima matre toxico impetus extinguitur, praefatus se ab eadem matre mortem accepisse, e qua vitam susceperat. Ayant ainsi effacé toute la responsabilité de Magnus Eriksson, il accompagne l'épisode d'une réflexion morale et d'une condamnation sans appel de Blanche :

Quelle femme vraiment infâme, plutôt originaire de l'extrême Barbarie que du royaume de France! Je lis en effet qu'elle fut la toute première à enseigner chez nous la fabrication des poisons. Mais nous devons à la bienveillance divine qu'il n'y ait eu jusqu'à aujourd'hui dans nos contrées pas de femmes, ou assurément qu'un nombre infime d'entre elles, pour l'imiter. ${ }^{135}$

Tout le projet de Johannes Magnus était de donner de l'histoire de ses ancêtres suédois l'image la plus glorieuse possible: la vénéneuse Blanche ne pouvait être qu'une étrangère, qui avait acclimaté, sans grand succès, les poisons en Suède. Mais remarquons qu'il reconnaît à peine ses origines françaises: peut-être souhaitait-il ménager la France, grand royaume catholique qu'il aurait été peu élégant de transformer en terre de poisons à l'heure où il espérait recouvrer le siège perdu d'Uppsala.

Dans la Scandinavie médiévale, les empoisonnements les plus redoutés relevaient certainement de l'intoxication accidentelle: dans un ouvrage publié en 1928, Ingvald Reichborn-Kjennerud rappelle que les paysans faisaient un signe de croix sur la bière afin d'écarter tout risque, soulignant au passage que cette pratique existait encore à son époque ${ }^{136}$. Mais le poison, arme criminelle, occupait comme dans le reste de l'Occident une place dans l'imaginaire des hommes. En Suède, le poison, conçu comme un maléfice, est évoqué dans quelques articles de loi qui ont révélé tout leur pouvoir au moment des grands procès en sorcellerie du XvII ${ }^{e}$ siècle. Au Moyen Âge, les accusations d'empoisonnement font partie des accessoires utilisés sur la scène politique, plus souvent que le poison ne l'est sans doute lui-même. Si dans certains cas, celui d'Inge ou des bûchers gotlandais de 1350, ce sont des hommes qui sont accusés ou exécutés, les 
femmes, dans les lois provinciales et la littérature, semblent plus souvent correspondre au portrait rêvé de l'empoisonneur, surtout si elles sont étrangères et peuvent cristalliser le ressentiment contre un peuple ennemi ou un adversaire politique. Le poison apparaît ainsi comme la métaphore des transgressions de la femme dans l'espace public, de ses aspirations, réelles ou supposées, à exercer un pouvoir.

La reine Blanche, d'origine française, fut ainsi victime dès le xIV siècle d'une rumeur qui mit longtemps à se dissiper. Au XIX siècle, August Strindberg s'empara du mythe. $\mathrm{Au}$ dernier acte de sa pièce La saga des Folkungar, dans laquelle il malmène quelque peu la chronologie, il met en scène le roi Magnus, Blanche et leur fils. Il fait mourir Erik de la peste et ne décrit donc pas Blanche en empoisonneuse. Cependant, sous sa plume, le venin reste féminin et Strindberg place malicieusement dans la bouche de sainte Brigitte ces mots qui décrivent la reine :

hos henne ligger gifter som hos blomman Bella Donna: $i$ ett skönt kärl, så att man icke vill tro det finns där ${ }^{137}$.

(en elle il y a du poison, comme dans la fleur de belladone, dans une enveloppe si belle qu'on ne veut pas croire qu'il est là).

\section{NOTES}

1. Kulturhistoriskt lexikon för nordisk medeltid (désormais KLNM),sous la dir. de J. Granlund et I. Andersson, Malmö, 1956-1978, vol. V, col. 296 (article giftplanter).

2. Sur ces pratiques, voir F.-X. Dillman, Les magiciens dans l'Islande ancienne. Études sur la représentation de la magie islandaise et de ses agents dans les sources littéraires norroises, Acta Academiae Regiae Gustavi Adolphi XCII, Uppsala, 2006.

3. Sagas islandaises, trad. de R. Boyer, Paris, 1987, Saga d'Egill, fils de Grímr le Chauve, p. 77.

4. Snorri Sturluson, Histoire des rois de Norvège, trad. de F.-X. Dillman, Paris, 2000, p. 161.

5. Saxo Grammaticus, La Geste des Danois, trad. de J.-P. Troadec, Paris, 1995, p. 391-392.

6. F. Collard, Le crime de poison au Moyen Âge, Paris, 2003, p. 134.

7. Id., Pouvoir et poison. Histoire d'un crime politique de l'Antiquité à nos jours, Paris, 2007, p. 194-208.

8. Les chroniques de la fin du Moyen Âge se souviennent encore du crime de Birger, la référence au banquet de Nyköping suffisant alors à résumer tout son règne : "Jak svälte mina bröder til död thy dreffs / jak aff rikit j nödh / J'ai fait mourir mes frères de faim, / c'est pourquoi j'ai été contraint de quitter le royaume. ", Svenska medeltids dikter och rim, éd. G. B. Klemming, Stockholm, 1882, p. 229, v. 379-380.

9. K. F. Söderwall, Studier öfver konunga-styrelsen, Lund, 1880, p. 39.

10. Um styrilsi konunga ok höfthinga, éd. L. Moberg, Stockholm, 1964, p. 61-62: Han takr til medh wålzgärningom ok illom rådhom / thera lif fördärua huilka ledh han förmå / medh förgärningom / förderuilsom / fängilsom / ok fulan dödh.

11. Magister Mathias, Homo conditus, éd. A. Piltz, Lund, 1984, p. 64.

12. B. Ankarloo, Trolldoms processerna i Sverige, Lund, 1971, p. 35.

13. K. F.Söderwall, Ordbok öfver svenska medeltids-språket, I, Lund, 1894-1918, p. 285 (sens 2) et Å. Holmbäck et E. Wessén, Magnus Erikssons Landslag i nusvensk tolkning, Lund, 1962, p. 221, n. 49. Par commodité, nous traduirons simplement, dans la suite de cet article, le terme forgärning par empoisonnement, bien qu'il ait une signification beaucoup plus large. 
14. C. J. Schlyter, Glossarium ad Corpus iuris Sueo-Gotorum antiqui, Corpus iuris sueo-gotorum antiqui. Samling af Sweriges gamla lagar, vol. XIII, Stockholm, 1877, p. 177 (article forgcerning) et p. 481 (article ogcerning). Voir aussi F.-X. Dillman, op. cit., p. 214.

15. Ce sont les termes que l'on trouve en particulier dans les ouvrages de médecine en ancien suédois. Voir KLNM,IV, col. 478-480 (article forgiftning).

16. C. J. Schlyter, Glossarium ad Corpus iuris Sueo-Gotorum antiqui, op. cit., p. 163 (article firigcera, sens 5). Le terme renvoie au verbe faire : forgärning, ou ogärning, signifie étymologiquement « une mauvaise action » et firigcera, « détruire ».

17. F.-X. Dillman, op. cit., p. 214 et C. J. Schlyter, op. cit., p. 177.

18. F. Collard, Le crime de poison au Moyen Âge, Paris, 2003, p. 24-32.

19. B. Ankarloo, op. cit., p. 36.

20. Certaines théories proposent une datation plus tardive. La présentation des lois suédoises dans toute leur complexité et l'évocation des multiples débats qu'elles ont suscités dépassent très largement le cadre de cet article. Pour un aperçu récent de ces enjeux et sur le fonctionnement de la justice en Suède, voir T. Lindkvist, "Droit et genèse de l'État dans la Suède médiévale : royauté et communautés ", Justice et législation, sous la dir. de A. Padoa-Schioppa, Paris, 2000, p. 251-270 et P. Line, Kingship ans State Formation in Sweden 1130-1290, Leiden-Boston, 2007, p. 151-174.

21. Cette loi a tout d'abord été considérée comme une ancienne loi de la province du Västmanland. Elsa Sjöholm y voit une loi du Värmland (E. Sjöholm, Sveriges medeltidslagar. Europeisk rättstradition i politisk omvandling, Lund, 1988, p. 321-329), mais cette hypothèse n'a pas été retenue.

22. Toutes les lois ont été publiées au XIX ${ }^{\mathrm{e}}$ siècle par H. S. Collin et C. J. Schlyter, Corpus iuris sueogotorum antiqui Samling af Sveriges gamla lagar, Lund-Stockholm, 1827-1877. C'est à cette édition qu'il sera fait référence. On trouve deux anciennes traductions en français par L. Beauchet, Loi de Westrogothie, Paris, 1894 et Id., Loi d'Upland, Paris, 1908.

23. Par commodité, nous lui donnerons le nom de Vådamålsbalk qui figure dans la traduction en suédois contemporain de la loi (Å. Holmbäck et E. Wessén, Svenska Landskapslagar, Ser.1, Ostgötalagen, Lund, 1933).

24. Le marc suédois correspondait à 210,6 grammes d'argent. Pour donner une comparaison, il suffit de dire que, dans la Loi de Magnus Eriksson, selon l'article XI du Code du roi, le prix d'un bon cheval de guerre, qui permet de servir le roi en vue d'obtenir une exemption d'impôt, est de 40 marcs, ce qui représente l'équivalent d'environ vingt vaches, soit presque six ans du salaire moyen d'un journalier.

25. C. J. Schlyter, op. cit., vol. V, Westmanna-lagen, Lund, 1841, p. 10.

26. La législation suédoise n'offre donc pas de réelle originalité par rapport à la manière dont le crime est traité ailleurs en Occident. Voir F. Collard, op. cit., 2003, p. 145 et suiv.

27. Å. Holmbäck et E. Wessén, Svenska Landskapslagar, Ser. 1, Ostgötalagen, Lund, 1933, p. 67.

28. C. J. Schlyter, op. cit., vol. III, Upplands-lagen, Stockholm, 1834, p. 151.

29. C. J. Schlyter, Glossarium ad Corpus iuris Sueo-Gotorum antiqui, op. cit., p. 444.

30. H. S. Collin et C. J. Schlyter, op. cit., vol. I, Westgöta-Lagen, p. 119 (article II § 9)

31. R. Hemmer, "De svenska medeltidslagarna stadganden om skadlig trolldom och förgifting ", Tidsskrift for rettsvitenskap, 1947, p. 409-446. Voir la critique de B. Ankarloo, op. cit., p. 35-36.

32. Béatrice Delaurenti, La puissance des mots « virtus verborum ». Débats doctrinaux sur le pouvoir des incantations au Moyen Âge, Paris, 2007.

33. Le hundare est une subdivision administrative et judiciaire d'une province. Le mot, utilisé seulement dans les provinces situées autour du lac Mälaren, est plus tard remplacé par le terme général härad, qui a la même signification, et qui correspond à ce qui, ailleurs en Occident, s'appelle une centaine. Le tribunal du härad est l'institution judiciaire de base dans la Suède 
médiévale qui ne connaît pas le système de la seigneurie. La plus haute instance judiciaire du royaume est constituée par les tribunaux royaux (räfter).

34. Le bonde est un homme libre, un paysan propriétaire de sa terre. Ce groupe possédait encore à la fin du Moyen Âge la majorité des terres du royaume de Suède et jouait un rôle politique non négligeable. Les lois provinciales étaient en grande partie rédigées du point de vue de ces paysans libres, mais l'influence grandissante de l'aristocratie et l'émergence de groupes privilégiés limitèrent peu à peu leur poids (Voir, par exemple, T. Lindkvist, " The peasantery and Peasant Communities ", Campagnes médiévales. L'Homme et son espace. Études offertes à Robert Fossier, sous la dir. d'É. Mornet, Paris, Publication de la Sorbonne, 1995, p. 387-398). L'ajout de la remarque finale s'explique par le fait que lier une personne innocente est passible d'un lourd châtiment (Å. Holmbäck et E. Wessén, Svenska Landskapslagar, Ser. 1, Upplandslagen, Lund, 1933, p. 125, n. 108). Cette disposition a probablement pour but d'encourager l'accusateur, qui ne risque rien même si la femme est finalement jugée innocente.

35. C. J. Schlyter, op. cit, vol. III, Upplands-lagen, Stockholm, 1834, p. 149-150.

36. Voir, par exemple, le châtiment réservé à une femme qui assassine son mari : C. J. Schlyter, Upplands-lagen, op. cit., p. 145.

37. C. J. Schlyter, Upplands-lagen, op. cit., p. 253-254.

38. Å. Holmbäck et E. Wessén, Svenska Landskapslagar, Ser.1, Upplandslagen, Lund, 1933, p. 125, n. 106.

39. C. J. Schlyter, op. cit., vol. VI, Bjärköa-rätten, Stockholm, 1844, p. 132 : Hwilikin mabar annæen myrpir. warpor til wnnin mep sex mannum. han skal stclles. celler vœri sik mep tolf mannæe epe. havin morpbrcernarce sama reet. Miipir konce nokon. hon skal quik i iorb grawces. $\$ 1$ hwar sum giwcer adrum forgauor mapcer celler konce. poem ma i bali brcennoe.

40. Ogilder signifie que des amendes ne seront pas exigées de la personne qui la tuera pour se venger (voir C. J. Schlyter, Glossarium ad Corpus iuris Sueo-Gotorum antiqui, op. cit., p. 480, sens 3).

41. H. S. Collin et C. J. Schlyter, Westgöta-lagen, op. cit., p. 22.

42. Ibid., p. 117.

43. Ibid., p. 153.

44. B. Ankarloo, op. cit., p. 32.

45. Å. Holmbäck et E. Wessén, Svenska Landskapslagar, Ser. 5, Yngre Västgötalagen, Lund, 1946, p. 301, n. 23.

46. Le prcetylter eper mentionné dans ce texte est un serment prêté par trois groupes de douze hommes qui jurent ensemble, ici pour prouver l'innocence de la femme (voir C. J. Schlyter, Glossarium ad Corpus iuris Sueo-Gotorum antiqui, op. cit., p. 658, sens 2 et p. 759).

47. H. S. Collin et C. J. Schlyter, op. cit., vol. II, Östgöta-lagen, Stockholm, 1830, p. 85.

48. C. J. Schlyter, op. cit., vol. X, Konung Magnus Erikssons lagen, Stockholm, 1862, p. 281-282.

49. C. J. Schlyter, Konung Magnus Erikssons lagen, op. cit., p. 276.

50. C. J. Schlyter, op. cit., vol. XI, Konung Magnus Erikssons stadslag, Stockholm, 1865, p. 323-324 et Ibid., vol. XII, Konung Christoffers lagen, Stockholm, 1869, p. 302.

51. C. J. Schlyter, Glossarium ad Corpus iuris Sueo-Gotorum antiqui, op. cit., p. 164.

52. H. S. Collin et C. J. Schlyter, Westgöta-lagen, op. cit., p. 38 : Iak sa at pu reet a quiggrindu lösharceb. ok $i$ trols ham ba alt var iamrist nat ok daghér. kallwer hance kunna firigcerce kono cellwer. ko. paet cer. vkuapins ord.

53. Ibid., p. 153.

54. Å. Holmbäck et E. Wessén, Svenska Landskapslagar, Ser. 5, Yngre Västgötalagen, op. cit., p. 301, n. 19.

55. C. J. Schlyter, Konung Magnus Erikssons stadslag, op. cit., p. 285.

56. Svenskt Diplomatarium från och med år 1401, t. III, éd. C. Silfverstolpe, Stockholm, 1885-1902, $\mathrm{n}^{\circ} 2386$, p. 256-257.

57. Ibid., n 2391, p. 260. 
58. Sur ces questions, voir la thèse que prépare actuellement Christine Ekholst à l'université de Stockholm, För varje brottsling ett straff. Föreställningar om kön $i$ den medeltida straffrätten. Je remercie ici Christine Ekholst de m'avoir permis de lire son étude inédite sur la construction du genre dans les dispositions des lois suédoises concernant la sorcellerie et l'empoisonnement (Genuskonstruktioner i de svenska medeltidslargarnas bestämmelser om trolldom och förgörning).

59. Cette exception n'est pas la seule. Voir C. Ekholst, «Defending One's Rights. Aspects of Violence, Honor and Gender in Swedish Medieval Law », Revue d'histoire nordique, 4, 2007, p. 68-81, en particulier p. 77-80.

60. H. S. Collin et C. J. Schlyter, Westgöta-lagen, op. cit., p. 55.

61. L'étude de François-Xavier Dillman, qui ne prend en compte que des sources littéraires norroises, semblent montrer qu'il n'en est rien (F.-X. Dillman, op. cit., p. 143-160). En dehors des lois, la rareté des sources ne permet guère de sonder aussi précisément l'imaginaire suédois.

62. F. Collard, op. cit., 2003, p. 111-117.

63. C. J. Schlyter, Westmanna-lagen, op. cit., p. 238.

64. La traduction par "empoisonne » est sans doute trop précise dans ce contexte. Le verbe signifie peut-être seulement «tuer ». L'article a été repris dans le même code dans la Nouvelle Loi $d u$ Västergötland (article XIX) et dans le Code des causes graves des deux lois nationales et de la Loi urbaine. C'est sa place dans ces lois qui incite à traduire par « empoisonner ». En effet, dans la Loi de Magnus Eriksson, l'article qui envisage la même situation est l'article VI du Code des causes graves: il suit l'article V qui, nous l'avons vu, évoque le cas où une personne «forgör (...) mep trulldom celle andrum forgerningum / tue (...) en usant de sorcellerie ou d'autres pratiques magiques ». Le fait que le verbe forgör soit aussi placé au début de l'article VI indique qu'il faut sans doute lui donner le même sens que dans l'article V. Comme le remarque Elias Wessén, le contexte de cet article correspond pourtant mieux aux articles II et III du code qui décrivent des infanticides (Å. Holmbäck et E. Wessén, Magnus Erikssons Landslag, op. cit., p. 219, n. 24). La même observation peut être faite dans la Loi urbaine et la Loi de Christophe de Bavière.

65. H. S. Collin et C. J. Schlyter, Westgöta-Lagen, op. cit., p. 29.

66. H. S. Collin et C. J. Schlyter, Östgöta-lagen, op. cit., p. 85.

67. C. J. Schlyter, Konung Magnus Erikssons lagen, op. cit., p. 276 : Forgör maper manne celle kunu, kona kunu cellæe manne, meb trulldom cellce andrum forgerningum, sua cet han celle hon faar döb af, miste liiv sit for polika gerning.

68. C. J. Schlyter, Konung Magnus Erikssons stadslag, op.cit., p.228-229: Bør kona ella man forgcerninga, ok weerdher bar a taken, tha skal man hona ella han mcedh forgcerningum taka ok a radztuw ledha.

69. C. J. Schlyter, Konung Magnus Erikssons lagen, op.cit., p. 276 ; Id., Konung Magnus Erikssons stadslag, op. cit., p. 324 et Id., Konung Christoffers lagen, op. cit., p. 302-303.

70. En Occident, le sorcier fait place à la sorcière à la fin du Moyen Âge et, comme l'a montré Bengt Ankarloo pour la Suède, les procès pour maléfices n'impliquent que $21 \%$ des hommes avant 1614 et $14 \%$, au plus fort des grands procès en sorcellerie, entre 1668 et 1675 . Voir B. Ankarloo, op. cit., p. 270.

71. M. Douglas, Comment pensent les institutions, Paris, 1999.

72. C. J. Schlyter, Konung Christoffers lagen, op. cit., p. 300-301.

73. A. Murray, Suicide in the Middle Ages, vol. 2, The Curse on Self Murder, Oxford, 2000, p. 33-34.

74. H. Munktell, "Två straffrätts historiska studier ", Uppsala Universitets årskrift, $1940: 5$, Uppsala, 1940, p. 7.

75. A. Murray, op. cit., p. 481-482.

76. Ces paroles, rapportées à chaque fois au style direct, s'adressent à la femme du paysan.

77. Cette phrase n'apparaît pas dans la Loi d'östergötland.

78. H. S. Collin et C. J. Schlyter, Östgöta-lagen, op. cit., p. 119-120. 
79. Å. Holmbäck et E. Wessén, Svenska Landskapslagar, Ser. 1, Ostgötalagen, Lund, 1933, p. 137, n. 18 et Id., Magnus Erikssons Landslag, Lund, 1962, p. 68, n. 24a.

80. H. S. Collin et C. J. Schlyter, Östgöta-lagen, op. cit., p. 85.

81. C'est le cas, rappelons-le, dans l'article XIX du Manhoelghis balker de la Loi d'Uppland.

82. H.Yrving, Gotlands Medeltid, Visby, 1978.

83. La Peste noire, arrivée dès 1347 à Gênes, n'atteignit la Scandinavie qu'à partir de 1349 . L'épidémie suivit trois routes pour arriver en Suède : du sud, la peste entra en Scanie dès 1349, puis au début de l'année 1350, elle pénétra par l'ouest de Norvège (où, à Oslo, la maladie avait éclaté dès février 1349). Enfin, elle se répandit aussi par les voies commerciales qui venaient d'Allemagne du nord en 1350. Voir O.J. Benedictow, "Plague», Medieval Scandinavia: An Encyclopedia, sous la dir. de P. Pulsiano, New-York-Londres, 1993, p. 508 et D. Harrison, Stora Döden. Den värsta katastrof som drabbat Europa, Stockholm, 2000, p. 381 et suiv.

84. Diplomatarium suecanum, t. VI, $\mathrm{n}^{\circ} 4655$, p. 259 : omnes puteos in civitatibus Stocholm, Arosie, Arboga et singulas paludes, aquas stantes, puteos alios, quo transiit Sweciam, circumquaque suis veneficiis intoxicavit.

85. Ibid., p. 259.

86. Ibid, p. 259 : nescio plura vobis dicere, sed tota Christianitas est per Judoeos et pessimos nos intoxicata.

87. KLNM VIII, col. 78, article «jøder »; J. N. Biraben, Les hommes et la peste en France et dans les pays méditerranéens, t. 1, Mouton-Paris-La Haye, 1975, p. 60 ; L. Musset, Les peuples scandinaves au Moyen Âge, Paris, 1951, p. 275 ; D. Harrison, Stora Döden, op. cit., p. 405-407.

88. Diplomatarium suecanum, t. VI, $\mathrm{n}^{\circ} 4655$, p. 259 : Item fatebatur [quod] feria secunda Penthecostes, cum missam celebra[re]t in ecclesia sancti Olavi nobiscum, mapulam, quam ad hoc officium peragendo habuit, ita ut omnes in offertorio ipsam osculantes tercia die fuerunt mortui aut quarta et similiter omnes ipsos visitantes.

89. D. Wase, «Kyrkorna i Visby. Några reflektioner rörande deras tillkomst, funktioner och församling ", Gotländst Arkiv, 1990, p. 29-52, en particulier p. 35-36.

90. Codex Diplomaticus Lubecensis, Lübeckisches Urkundenbuch, t. I, Urkundenbuch der Stadt Lübeck, vol. 3, Lübeck, 1871, n 110.

91. I. Lindqvist, «De tre krönikorna om lagmän, kungar och biskopar. VgL IV. 14, 15 och 16. », Västgötalagen litterära bilagor - Medeltida svensk småberättelsekonst på poesi och prosa, Lund, 1941, p. 42 : Niundi war Ingi kononger, brodher Philipuscer konongs. Oc heter aptir Ingæe kononge, Halstens konongs brødhœr. Hanum war firi giort mæd ondom dryk $i$ Østrcegøtlandi, oc fek aff py bance. Æn Sweriki for e wol, moedhon per frondlingor roedhu.

92. P. Line, op. cit., p. 590.

93. Ibid., p. 42.

94. "Hii reges christiani et hoc ordine rexerant in swecia quo ponuntur", Småstycken på fornsvenska, éd. G. E. Klemming, Stockholm, 1868-1881, éd. p. 268 : Nono loco frater philippi ingo iunior Jngonis prioris seu patrui suis nomen sortitus regnum accepit qui postmodum in osgocia wretis inpocionatus decessit.

95. N. Ahnlund, « Vreta kloster äldsta donatorer », Historisk Tidskrift, 1945, p. 301-351.

96. Idid., p. 303 et «Catalogus regum Sveciæ paganorum pariter et christianorum ab Ingone ad Ericus Pomeranum », éd. E. M. Fant, Scriptores rerem svecicanum medii aevii, t. I, Uppsala, 1818, p. 15 , n. i.

97. «Lilla Rim-Krönikan », Svenska medeltids dikter och rim, éd. G. B. Klemming, Stockholm, 1882, p. 226.

98. Ericus Olai, Chronica regni Gothorum, éd. J. Öberg et E. Heuman, Stockholm, 1993, p. 63 (§ XIII, 43-44).

99. Olaus Petri, En swensk cröneka, éd. J. Sahlgren, Uppsala, 1917 : Och bleeff konung Inge forgiffuen $i$ Wreta $i$ Östergötland, aff them, til ewentyr, som icke fingo göra så mykit ondt som the wille, ty han 
pläghade straffa them som orett giorde, kan och wel henda at noghro haffua warit i rådhet med som sielffue wille wara i regementit.

100. Ibid. : Segher och then Danska Crönekan (...) at en hest slipade thenna konung Inga til dödz ther han hengde med foten $i$ böghlone. Men wora Swenska Cröneker seya endrechteliga at han wardt förgiffuin $i$ Wreta (...).

101. Johannes Magnus, Historia de omnibus Gothorum Sveorumque regibus, Rome, 1554, p. 588.

102. Svenskt Biografikt Lexikon, Stockholm, 1918-, vol. XIX, p. 781.

103. Pour le texte original, voir Erikskrönikan, enligt Cod; Holm. D2 jämte avvikande läsarter ur andra handskrifter, éd.R. Pipping, Stockholm, 1963 et, pour la traduction française, Erikskrönika, Chronique d'Éric, première chronique rimée suédoise, introduction, traduction, et commentaires C. Péneau, Paris, Publications de la Sorbonne, 2005, v. 272 à 283.

104. Ibid., v. 292-361.

105. F. Collard, op. cit., 2003, p. 172.

106. Svenska Medeltidens rim-krönikor, III - Nya krönikans forsättningar eller Sture-krönikorna, éd. G. E. Klemming, Stockholm, 1867-1868, p. 85, v. 2476-2477.

107. Ibid., p. 85-86, v. 2481-2491 : jezabell monde alla hänne kalla / mz rätta bör henne ey längher liffua / hon wiille sin son junkar hanis förgiffua / $m z$ eeth hönss som steektes full wäll /holkith hon gör wthan skäll / gud gaff honum thz tiil snille / thz han tha ekke foor ille / thz är ekke nw mynne / faar hon ekke annath synne / och faar hon siälff raadha / hon aktar komma swerige i waadha.

108. K. Hagnell, Sturekrönikan 1452-1496-Studier över en rimkrönikas tillkomst och sanningsvärde, Lund, 1941, p. 42-43.

109. Ibid., p. 74.

110. G. Carlsson, «Drottning Dorotheas svenska morgongåfva », Historisk tidskrift, 1911, p. 238-268 ; Sven-Erik Pernler, Sveriges kyrkohistoria. t. 2 : Hög-och senmedeltid, Stockholm, 1999, p. 171-175 et K. G. Lundholm, Sten Sture den äldre och stormännen, Lund, 1956, p. 111-112.

111. L. Musset, op. cit., p. 312.

112. Svenska Medeltidens rim-krönikor, III - Nya krönikans forsättningar eller Sture-krönikorna, éd. cit., p. $188-189$.

113. "Tilläg till Lilla Rim-Krönikan», Svenska medeltids dikter och rim, éd. G. B. Klemming, Stockholm, 1882, p. 238 et 243.

114. Olaus Petri, En swensk cröneka, éd. cit., p. 267.

115. G. Carlsson, Hemming Gadh, Uppsala, 1915, p. 100.

116. J. Svanberg, Sankt Göran och draken, Stockholm, 1993, p. 61-67.

117. Sancta Birgitta, Revelaciones. Book VIII, éd. H. Aili, Stockholm, 2001, p. 188, Révélation 48 (§ 95-96).

118. Ibid., p. 196, Révélation 48 ( $\$ 168-169)$ : Tercio vidisti, quod rege excogitante imponere in regno nouas exacciones tributorum, infusum fuit in manibus eius venenum. Significat quod opera regis regebantur dyabolico spiritu et prauis suggestionibus. Nam sicut venenum facit inquietacionem et infrigidacionem in corpore, sic rex sollicitabatur et inquietabatur malignis suggestionibus et cogitacionibus, scilicet inuestigando modos, quomodo obtineret possessiones et bona aliorum et aurum viatorum.

119. Sur cette révélation, voir aussi C. Péneau, «Révélations et élections. Le corps du roi et la parole dans les Révélations de sainte Brigitte ", Médiévales, 50, 2006, p. 77-102.

120. Sancta Birgitta, Revelaciones. Book VIII, éd. cit., p. 194, Révélation 48 (§ 148) : Coniungamus eciam nos tribus funibus. Primus funis sit in medio, quo vmbelicus tuus et meus coniungantur simul, quatinus me spirante trahas in te venenum meum, te vero spirante ego attraham in me interiora tua.

121. Sancta Birgitta, Revelaciones Extravagantes, éd. L. Hollman, Uppsala, 1956, Extravagante 59 : Tempore, quo beata Birgitta fuit magistra regine Blanche, quondam regine Suecie...

122. Sancta Birgitta, Revelaciones. Book VIII, éd. cit., Révélation 31.

123. Svenskt Biografikt Lexikon, Stockholm, 1918, t. III, p. 185-186. 
124. L. Sjöstedt, Krisen inom det svensk-skånska väldet 1356-1359, Lund, 1954, p. 165-215.

125. Voir S.Kraft, «En pamflett mot Magnus Eriksson i dess idépolitiska och litterära miljö », Historisk Tidskrift, 1927, p. 4 et S. Axelson, « Notiserna om Magnus Eriksssons kröning 1336 och hans nederlag vid Gata 1365 », Historisk Tidskrift, 1976, p. 184-188. Olle Ferm a proposé le nom d'un laïc, Karl Ulfsson, le grand-père du roi Karl Knutsson, qui fit des études à Paris et qui était un opposant à Magnus Eriksson : voir O. Ferm, Olaus Petri och Heliga Birgitta, Synpunkter på ett nytt sätt att skriva historia i 1500-talets Sverige, Stockholm, 2007, p. 18, n. 19.

126. I. Andersson, Källstudier till Sveriges historia 1230-1436. Inhemska berättande källor jämte Libellus Magnipolensis, Lund, 1928, p. 152-153.

127. "Libellus de Magno Erici Rege", Scriptores rerum suecicarum medii cevi, vol. III, éd. C. Annerstedt, Uppsala, 1871-1876, p. 14.

128. H. T.Gilkær, The Political Ideas of St.Birgitta and her Spanish Confessor, Alfonso Pecha. Liber Celestis Imperatoris ad Reges : A Mirror of Princes, Odense, 1993, p. 197-218. La perte de la Scanie fait partie des arguments que Brigitte donne aux chevaliers suédois dans la révélation où elle les encourage à prendre le pouvoir en Suède. La lettre a été publiée par B. Högman, Heliga Birgittas Originaltexter, Uppsala, 1951, p. 78-83. Voir également O. Ferm, «Heliga Birgitta program för uppror mot Magnus Eriksson - En studie i politisk argumentationkonst», Heliga Birgitta,Budskapet och förebilden - Föredrag vid jubileumssymposiet $i$ Vadstena 3-7 oktober 1991, sous la dir. d'A. Härdelin et M. Lindgren, Stockholm, 1993, p. 125-143.

129. "Libellus de Magno Erici Rege », éd. cit., p.13. Voir également C. Péneau, "Um styrilsi konunga ok höfpinga, un miroir inspiré de Gilles de Rome dans la Suède de la première moitié du $\mathrm{XIV}^{\mathrm{e}}$ siècle ", Le Prince au miroir de la littérature politique de l'Antiquité aux Lumières, sous la dir. de F. Lachaud et L. Scordia, Rouen, 2007, p. 200.

130. F. Collard, op. cit., 2003, p. 116.

131. "Sammanfogningen mellan Gamla och Nya Krönikan », Svenska Medeltidens rim-krönikor, IGamla eller Eriks-krönikan, éd.G.E. Klemming, Stockholm, 1865, p. 178-179, v. 223-230.

132. Ericus Olai, Chronica regni Gothorum, éd. cit., p. 130 (§XXXV, 25-26).

133. Voir O. Ferm, op. cit., p. 19-23.

134. Olaus Petri, En swensk cröneka, éd. cit., p. 118.

135. Johannes Magnus, Historia de omnibus Gothorum Sveorumque regibus, Rome, 1554, p. 670 : Infamis profecto mulier, quce potius ab extrema barbarie, quam a regno Gallice illuc peruenisset. Hanc enim omnium primam lego, quce venena apud nostros miscere docuisset. Sed diuince benegnitati debemus, quod nullas, aut certe paucissimas hactenus habuerit in nostris terris imitatrices.

136. I. Reichborn-Kjennerud, Vår gamle trolldomsmedisin, Oslo, 1928, p. 90. Le geste est attesté dans les sagas, en particulier dans la Saga d'Egill, fils de Grímr le Chauve évoquée en introduction. 137. A. Strindberg, Folkungasaga, Samlade skrifter, vol. 31, Stockholm, 1915, p. 126.

\section{RÉSUMÉS}

Les poisons ne furent pas les armes les plus utilisées dans la Suède médiévale. Cependant, les chroniques et les lois, plus que tout autre type de sources, proposent quelques cas intéressants relatifs à l'usage des poisons. Comme dans d'autres régions d'Europe, cet usage est lié à la magie et considéré comme un des pires crimes possibles. Dans les lois suédoises, la mort est le châtiment courant pour ces crimes dont les victimes peuvent être des hommes, des femmes, mais 
aussi des animaux. Dans les lois provinciales, les femmes seules sont décrites en empoisonneuses, mais dans les lois urbaines et nationales, hommes et femmes peuvent être considérés comme des manipulateurs de poisons. Dans l'historiographie, trois femmes sont accusées d'être des empoisonneuses. Le premier exemple peut être trouvé dans un texte inspiré par sainte Brigitte : Blanche de Namur, la femme du roi Magnus Eriksson, fut accusée d'avoir empoisonné son propre fils Erik. Les deux autres furent des reines danoises. À la fin du Moyen Âge, les reines étrangères apparaissaient en effet comme de véritables poisons au sein ducorps politique.

Poisons do not seem to be the most frequently used weapons in medieval Sweden. Nevertheless, more often than other types of sources, chronicles and laws offer some interesting examples of poisonings. As in other parts of Europe, the use of poison is linked with magic and is considered one of the worst possible crimes. In the books of Swedish law, death is the typical punishment for this crime whose victims can be men, women or even animals. In provincial laws, women are the only murderers who resort to poison, but in urban and national laws, men and women are both considered as possible poisoners. In literature, three women are blamed for killing people with poisonous potions. The first example is found in a text inspired by Saint Bridget: Blanche of Namur, king Magnus Eriksson's wife, was accused of having murdered her own son Erik. The two others were Danish queens. Towards the end of the Middle Ages, foreign queens became largely considered as being real poison inside the body politic.

\section{AUTEUR}

\section{CORINNE PÉNEAU}

Université Paris-XII Val-de-Marne. 